\title{
Doubly Hybrid Functionals Close to Chemical Accuracy for both \\ Finite and Extended Systems: Implementation and Test of XYG3 and XYGJ-OS
}

Yizhen Wang ${ }^{\mathrm{a},}$, Yajing $\mathrm{Li}^{\mathrm{a}}{ }^{\text {ฯ }}$, Jun Chen ${ }^{\mathrm{b}}$, Igor Ying Zhang*,a and Xin $\mathrm{Xu}^{*}$, a

${ }^{a}$ Collaborative Innovation Center of Chemistry for Energy Materials, Shanghai, Key Laboratory of Molecular Catalysis and Innovative Materials, MOE Key Laboratory of Computational Physical Sciences, Shanghai Key Laboratory of Bioactive Small Molecules, Department of Chemistry, Fudan University, Shanghai 200433, People's Republic of China

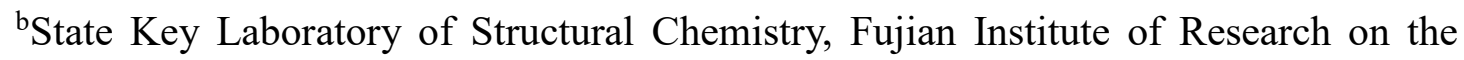
Structure of Matter, Chinese Academy of Sciences, Fuzhou 350002, People's

Republic of China

"Contributed equally to this work

*E-mail: igor_zhangying@,fudan.edu.cn; xxchem@fudan.edu.cn

1. Computational Details s2-s6

2. Reference s6-s8

$\begin{array}{ll}\text { 3. Tables } & \text { s9-s27 }\end{array}$

$\begin{array}{lr}\text { 4. Optimized Cartesian coordinates } & \text { s28-s47 }\end{array}$ 


\section{Computational Details}

\section{Main-group cubic crystals}

The calculations in this work were all performed using the FHI-aims package in the numerical integration framework. ${ }^{1}$ The atomic zeroth-order regular approximation (ZORA) was employed in the calculations to properly consider the effect of near-nuclear relativity on valenceand semicore-like electrons. For main-group cubic crystals investigated in this work, we employed the numerical atom-centered orbital (NAO) basis sets with valence-correlation consistency (NAO-VCC- $n \mathrm{Z}$ with $n=3,4,5) .{ }^{2}$ For (semi-)local and hybrid density functional approximations (DFAs), including BLYP, ${ }^{3-5}$ B3LYP, ${ }^{3-4,6-7} \mathrm{M} 06-\mathrm{L},{ }^{8} \mathrm{M} 06,{ }^{9} \mathrm{PBE},{ }^{10} \mathrm{PBE} 0,{ }^{11} \mathrm{SCAN},{ }^{12}$ and SCAN0 ${ }^{13}$ (using the same hybrid formula and parameters of PBE0, but replace PBE exchange and correlation by the corresponding SCAN ones), the calculations were performed with the NAO-VCC-3Z basis sets; while those of XYG3 ${ }^{14}$ and XYGJ-OS ${ }^{15}$ were obtained by the two-point complete extrapolation from NAO-VCC-2Z and $3 \mathrm{Z}(\mathrm{CBS}[2,3])$ :

$$
E[\infty]=\frac{E[2] 3^{3}-E[2] 2^{3}}{3^{3}-2^{3}}
$$

where $\mathrm{E}[n]$ is the XYG3 or XYGJ-OS total energy per atom using NAO-VCC-nZ basis sets (with $\mathrm{n}=2$ and 3).

The calculations with (semi-)local and hybrid density functional approximations (DFAs) were all performed in an all-electron/full potential framework, while the frozen-core approximation was used for the calculations of XYG3 and XYGJ-OS. For most of the main-group cubic crystals, only the electrons on the first valence shell were considered, however, for the element of $\mathrm{Na}$ in $\mathrm{NaF}$ and $\mathrm{NaCl}$, excluding the electrons on the occupied $2 \mathrm{~s}$ and $2 \mathrm{p}$ orbitals in the evaluation of the PT2 correlation would result in unphysical equation of state for $\mathrm{NaF}$ and $\mathrm{NaCl}$, because these orbitals are deeply involved to form the ionic bonds. In this work, the electron on the $2 \mathrm{~s}$ and $2 \mathrm{p}$ orbitals of $\mathrm{Na}$ were also considered for $\mathrm{NaF}$ and $\mathrm{NaCl}$. In consequence, the core basis functions from Gaussian-type cc-pCVnZ (with $n=2$ and 3$)^{16}$ were introduced to properly describe the PT2 correlation contribution from these inner-shell orbitals.

Table S1 lists the k-grid setting used for different DFAs with either Gamma-Center (GC) or 
Monkhorst-Pack (MP) sampling strategies. The two-point complete k-mesh extrapolation utilized for XYG3 and XYGJ-OS is:

$$
\begin{gathered}
\Delta E_{\text {total }}\left[n_{k}\right]=\frac{A}{n_{k}^{\alpha}} \\
\log \left(\Delta E_{\text {total }}\left[n_{k}\right]\right)=\log (\mathrm{A})-\alpha \log \left(n_{k}\right),
\end{gathered}
$$

where $n_{k}$ is the number of $\mathrm{k}$ points in each direction $\left(n_{k} \times n_{k} \times n_{k}\right)$. The exponential ' $\alpha$ ' determines the speed of the k-point convergence of different methods. For XYG3 and XYGJ-OS, $\alpha=3.0$ is used, which has been successfully used to extrapolated MP2 and RPA cohesive energies in previous studies.

For XYG3 and XYGJ-OS, the calculations of NAO-VCC-2Z and $3 Z$ with $6 \times 6 \times 6$ were carried out directly, while the energies of NAO-VCC-3Z with $8 \times 8 \times 8,\left(E_{\text {total }}^{N 3 Z}\left[n_{k}=8\right]\right)$, were obtained by using the down-sampling concept ${ }^{17}$ :

$$
E_{\text {total }}^{N 3 Z}\left[n_{k}=8\right]=E_{\text {total }}^{N 3 Z}\left[n_{k}=6\right]+\Delta E_{\text {total }}^{N 2 Z}\left[n_{k}=8\right]
$$

where $\Delta E_{\text {total }}^{N 2 Z}\left[n_{k}=8\right]$ is the energy change between $6 \times 6 \times 6$ and $8 \times 8 \times 8 \mathrm{k}$-points with the NAO-VCC-2Z basis set:

$$
\Delta E_{\text {total }}^{N 2 Z}\left[n_{k}=8\right]=E_{\text {total }}^{N 2 Z}\left[n_{k}=8\right]-E_{\text {total }}^{N 2 Z}\left[n_{k}=6\right] .
$$

To find the optimized lattice parameters for each main-group cubic material and each method, we calculated seven points within a range of $\pm 5 \%$ around the initial value of the lattice constant, and fited the respective (volume, energy) data points to the Birch-Murnaghan equation of state to obtain the optimized lattice constant. If all 7 lattice constants used to generate the equation of state lie within a range of $\pm 7 \%$ around the optimized value, the value is taken as the final, optimized lattice constant. Otherwise, this optimized lattice constant is used as a new starting point and more (volume, energy) data points are calculated, so that finally all materials' optimized lattice constants obtained from an equation of state fitted to seven points with lattice constant values in a range of $\pm 5 \%$ to $\pm 7 \%$ around the final, optimized lattice constant.

More details about the numerical convergence with respect to the basis set, k-grid and geometry optimizations have been provided in Reference 17 . We attached at the end of SI the geometries of each material in the FHI-aims format; while all the calculated cohesive properties by different methods are listed in Tables S2-4. For comparison, we also list MP2 and RPA results 
in the relevant tables, which were collected from Reference 17.

\section{Rutile and Anatase TiO2}

All the calculations of $\mathrm{TiO} 2$ crystals used the standard NAO basis sets with tight setting in FHI-aims. ${ }^{1}$ The atomic zeroth-order regular approximation (ZORA) was employed in all calculations to properly consider the effect of near-nuclear relativity on valence- and semicore-like electrons. Frozen-core approximation was used for the calculations of XYG3 and XYGJ-OS. The electrons on the occupied orbitals of $3 \mathrm{~s}, 3 \mathrm{p}, 3 \mathrm{~d}$, and $4 \mathrm{~s}$ are included for the $3 \mathrm{~d}$-block transition metal of Ti. The k-grid settings used for different DFAs are also listed in Table S1. Note that, in the calculations of XYG3 and XYGJ-OS, we used only $4 \mathrm{x} 4 \mathrm{x} 6$ for rutile and $4 \mathrm{x} 4 \mathrm{x} 4$ for anatase $\mathrm{TiO} 2$, which is almost touching the limit of our computational capability. Although the k-mesh incompleteness error and the basis-set incompleteness error might be large for the absolute cohesive energies, it has been argued to be negligible for the calculations of geometry parameters and also the relative energies ${ }^{17}$, which are the materials properties of interest in this work.

For the geometry optimization of rutile and anatase $\mathrm{TiO} 2$ calculations, we consider a series of unit cell volumes, for each of which all internal coordinates and the shape of the unit cell are fully relaxed; the total energy at the equilibrium structure and the lattice parameters are then obtained by fitting the Birch-Murnaghan equation of state in the same manner as we did for main-group cubic crystals mentioned above. For XYG3, XYGJ-OS and other hybrid DFAs, including B3LYP, SCAN0, and M06, the lattice parameters are determined by calculating the total DFA@X energies at different unit cell volumes with the crystal structures optimized by the DFA of $X .{ }^{18}$ In this work, we investigate the influence of different "X" methods, including PBE and SCAN, on the final SCAN0, XYG3, and XYGJ-OS results. As shown in Table S5, such kind of influence is negligible. Given that SCAN provides better lattice parameters than PBE, we present the XYG3 and XYGJ-OS results based on the SCAN geometries in the main text.

\section{2 bond dissociation energies}

For the molecular test set with 142 bond dissociation energies, Bond142, we refer interested authors to the reference of 19 for both computation details and the input geometries, but provides all the calculated results in Table S6. Briefly speaking, 92 bond dissociation reactions in Bond142 are established by 27 radicals and 76 molecules from the G3/99 set $^{20}$, while the rest 50 bond 
dissociation reactions need 5 more radicals and 39 more molecules that go beyond the G3/99 set. The bond types include C-H, $X-\mathrm{H}, \mathrm{C}-\mathrm{C}, \mathrm{C}-\mathrm{O}, \mathrm{C}-\mathrm{N}, \mathrm{C}-\mathrm{F}, \mathrm{C}-\mathrm{Cl}, \mathrm{C}-\mathrm{S}$, and $X-Y(X, Y \neq \mathrm{C}, \mathrm{H})$. The bond dissociation energies are derived from the standard heats of formation at $298 \mathrm{~K}$ of the corresponding radicals and molecules in gas phase. The heats of formation are calculated based on the theoretical atomization enthalpy using the NAO-VCC-4Z basis set and the frozen-core approximation in the computational platform of FHI-aims, ${ }^{1}$ which are corrected by the experimental atomization enthalpies of the constituent elements in their standard states at $298 \mathrm{~K}$.

\section{$\mathrm{CO}$ adsorption on a ragid $\mathrm{NaCl}(100)$ surface}

The $\mathrm{NaCl}(100)$ surface was modeled by a rigid two-layer slab with the experimental lattice of $5.64 \AA$, while the $\mathrm{CO}$ overlayer was simulated in $\mathrm{p}(1 \mathrm{x} 1)$ surface unit cell, corresponding to 1 monolayer (ML) coverage. The vacuum space between the periodic slabs in the Z-direction was set to $30 \AA$. A Gamma-center k-points grid mesh of $3 \times 3 \times 1$ was used. The convergence properties of slab layers and k-points at the PBE level of theory has been carefully examined in the previous literature. ${ }^{21} \mathrm{NAO}-\mathrm{VCC}-3 \mathrm{Z}$ basis set was employed for all calculations Note that the self-consistent convergence of SCAN0 calculations failed for the geometry optimization of C-down adsorption configuration. The calculations of XYG3 and XYGJ-OS used NAO-VCC-3Z for the geometry optimization and frequency evaluations, while the adsorption energies were extrapolated to the complete-basis-set limit with NAO-VCC-3Z and 4Z (Eq S1). Frozen-core approximation was employed. The electrons on the occupied orbitals of $2 \mathrm{~s}, 2 \mathrm{p}$, and $3 \mathrm{~s}$ are included for $\mathrm{Na}$, while for all other elements, including $\mathrm{C}, \mathrm{O}$, and $\mathrm{Cl}$, only the electrons on the first valance shell are considered. In consequence, the core basis functions from Gaussian-type cc-pCVnZ (with $\mathrm{n}=2$ and $3)^{16}$ were introduced on top of NAO-VCC-nZ $(n=3,4)$ for Na only, which is important to properly describe the PT2 correlation contribution from the inner-shell orbitals of $2 \mathrm{~s}$ and $2 \mathrm{p}$.

Finite-displacement strategy was employed to performance the geometry optimization. The displacement is set to $0.001 \AA$. The convergence criterion of the largest remaining force on each atom is set to $0.02 \mathrm{eV} \AA^{-1}$. Table $\mathrm{S} 8$ provides the optimized $\mathrm{dZ}$ of PBE, PBE0, and M06 methods by using the numerical force and using the analytic force, indicating that the geometry error due to the use of numerical force can be smaller than $0.01 \AA$ in this case. Note that the geometry 
optimization of SCAN using analytic force fails in this test.

The adsorption energy $E_{a}$ is defined as the energy difference of the C-down configuration (Geom6 on Page S46) with respect to the free CO monolayer in the same unit cell (Geom8 on Page S46) and the two-layer rigid $\mathrm{NaCl}(100)$ slab (Geom9 on Page S47):

$$
E_{a}=E[\mathrm{C}-\mathrm{down}]-E[\mathrm{CO}]-E[\mathrm{NaCl}]
$$

It is worth to point out that we use a free CO monolayer in the same unit cell (Geom8 on Page $\mathrm{S} 45)$, but not a free $\mathrm{CO}$ in the above definition. It is because the experimental $\mathrm{CO}$ adsorption energy of $-18 \mathrm{~kJ} / \mathrm{mol}^{22}$ and accurate QM/MM results of $-17.8 \mathrm{kcal} / \mathrm{mol}^{23}$ was obtained with low $\mathrm{CO}$ coverage. In this work, the $\mathrm{CO}$ adsorption is simulated in $\mathrm{p}(1 \times 1)$ surface unit cells, corresponding to 1 monolayer (Geom6 for C-down configuration and Geom7 for O-down configuration). The calculations with larger unit cells for lower CO coverage are currently unaffordable for XYG3 and XYGJ-OS. In order to remove the undesired CO-CO lateral interaction, we take a free CO monolayer in the same unit cell (Geom8 on Page S46), but not a free $\mathrm{CO}$ as the reference. Basis set influence on the XYGJ-OS adsorption energy has been investigated and reported in Table S10. Comparing to the complete-basis-set limit results (-18.9 $\mathrm{kJ} / \mathrm{mol}$ ) The basis-set error with NAO-VCC-3Z is about $2.7 \mathrm{~kJ} / \mathrm{mol}$, which is about $15 \%$ of the experimental number and is apparently not negligible. The XYG3 result reported in this work is its NAO-VCC-3Z value corrected by the XYGJ-OS value of $2.7 \mathrm{~kJ} / \mathrm{mol}$.

Based on the optimized geometries, we also performed the $\mathrm{CO}$ vibration frequency calculations. The displacement is set to $0.013 \AA$. Table S9 investigates the influence of displacement on the final frequency of PBE method, which can be less than $3 \mathrm{~cm}^{-1}$. Table S9 suggests that the deviation of our PBE frequency is only about $2 \mathrm{~cm}^{-1}$ with respect to the number collected from the recent paper $^{21}$, which was obtained by using completely different plane-wave basis and based on analytic forces, demonstrating the robustness of our energy-based frequency evaluation for the $\mathrm{CO}$ vibration. The frequency shift was defined as the $\mathrm{CO}$ vibrational frequencies on C-down (Geom6 on Page S46) or O-down (Geom7 on Page S46) configuration to that of free CO monolayer (Geom8 on Page S46).

\section{Reference}


1. Blum, V.; Gehrke, R.; Hanke, F.; Havu, P.; Havu, V.; Ren, X.; Reuter, K.; Scheffler, M., Ab initio molecular simulations with numeric atom-centered orbitals. Comput. Phys. Commun. 2009, 180 (11), 2175-2196.

2. Zhang, I. Y.; Ren, X.; Rinke, P.; Blum, V.; Scheffler, M., Numeric atom-centered-orbital basis sets with valence-correlation consistency from H to Ar. New Journal of Physics 2013, 15 (12), 123033.

3. Becke, A. D., Density-functional exchange-energy approximation with correct asymptotic-behavior. Phys. Rev. A 1988, 38 (6), 3098-3100.

4. Lee, C. T.; Yang, W. T.; Parr, R. G., Development of the colle-salvetti correlation-energy formula into a functional of the electron-density. Phys. Rev. B 1988, 37 (2), 785-789.

5. Miehlich, B.; Savin, A.; Stoll, H.; Preuss, H., RESULTS OBTAINED WITH THE CORRELATION-ENERGY DENSITY FUNCTIONALS OF BECKE AND LEE, YANG AND PARR. Chem. Phys. Lett. 1989, 157 (3), 200-206.

6. Becke, A. D., Density - functional thermochemistry. III. The role of exact exchange. J. Chem. Phys. 1993, 98 (7), 5648-5652.

7. Stephens, P. J.; Devlin, F. J.; Chabalowski, C. F.; Frisch, M. J., Ab Initio Calculation of Vibrational Absorption and Circular Dichroism Spectra Using Density Functional Force Fields. J. Phys. Chem. 1994, 98 (45), 11623-11627.

8. Zhao, Y.; Truhlar, D. G., A new local density functional for main-group thermochemistry, transition metal bonding, thermochemical kinetics, and noncovalent interactions. J. Chem. Phys. 2006, 125 (19), 194101.

9. Zhao, Y.; Truhlar, D. G., The M06 suite of density functionals for main group thermochemistry, thermochemical kinetics, noncovalent interactions, excited states, and transition elements: two new functionals and systematic testing of four M06-class functionals and 12 other functionals. Theor. Chem. Acc. 2008, 120 (1-3), 215-241.

10. Perdew, J. P.; Burke, K.; Ernzerhof, M., Generalized gradient approximation made simple. Phys. Rev. Lett. 1996, 77 (18), 3865-3868.

11. Adamo, C.; Barone, V., Toward reliable density functional methods without adjustable parameters: The PBE0 model. J. Chem. Phys. 1999, 110 (13), 6158-6170.

12. Sun, J.; Ruzsinszky, A.; Perdew, J. P., Strongly Constrained and Appropriately Normed Semilocal Density Functional. Phys. Rev. Lett. 2015, 115 (3), 036402.

13. Hui, K.; Chai, J.-D., SCAN-based hybrid and double-hybrid density functionals from models without fitted parameters. The Journal of Chemical Physics 2016, 144 (4), 044114.

14. Zhang, Y.; Xu, X.; Goddard, W. A., Doubly hybrid density functional for accurate descriptions of nonbond interactions, thermochemistry, and thermochemical kinetics. Proc. Natl. Acad. Sci. U. S. A. 2009, 106 (13), 4963-4968.

15. Zhang, I. Y.; Xu, X.; Jung, Y.; Goddard, W. A., A fast doubly hybrid density functional method close to chemical accuracy using a local opposite spin ansatz. Proc. Natl. Acad. Sci. U. S. A. 2011, 108 (50), 19896-19900.

16. Pritchard, B. P.; Altarawy, D.; Didier, B.; Gibson, T. D.; Windus, T. L., New Basis Set Exchange: An Open, Up-to-Date Resource for the Molecular Sciences Community. Journal of Chemical Information and Modeling 2019, 59 (11), 4814-4820.

17. Zhang, I. Y.; Logsdail, A. J.; Ren, X.; Levchenko, S. V.; Ghiringhelli, L.; Scheffler, M., Main-group test set for materials science and engineering with user-friendly graphical tools for error analysis: systematic benchmark of the numerical and intrinsic errors in state-of-the-art 
electronic-structure approximations. New Journal of Physics 2019, 21 (1), 013025.

18. Cui, Z.-H.; Wu, F.; Jiang, H., First-principles study of relative stability of rutile and anatase $\mathrm{TiO} 2$ using the random phase approximation. Physical Chemistry Chemical Physics 2016, 18 (43), 29914-29922.

19. Wu, J. M.; Xu, X., Improving the B3LYP bond energies by using the X1 method. Journal of Chemistry Physics 2008, 129 (16), 164103-11.

20. Curtiss, L. A.; Raghavachari, K.; Redfern, P. C.; Pople, J. A., Assessment of Gaussian-3 and density functional theories for a larger experimental test set. J. Chem. Phys. 2000, 112 (17), 7374-7383. 21. Chen, J.; Hariharan, S.; Meyer, J.; Guo, H., Potential Energy Landscape of CO Adsorbates on $\mathrm{NaCl}(100)$ and Implications in Isomerization of Vibrationally Excited CO. The Journal of Physical Chemistry C 2020, 124 (35), 19146-19156.

22. Richardson, H. H.; Baumann, C.; Ewing, G. E., Infrared spectroscopy and thermodynamic measurements of $\mathrm{CO}$ on $\mathrm{NaCl}$ films. Surface Science 1987, 185 (1), 15-35.

23. Vogt, J.; Vogt, B., The structure of carbon monoxide adsorbed on the $\mathrm{NaCl}(100)$ surface- $\mathrm{A}$ combined LEED and DFT-D/vdW-DF study. The Journal of Chemical Physics 2014, 141 (21), 214708.

24. Tran, F.; Stelzl, J.; Blaha, P., Rungs 1 to 4 of DFT Jacob's ladder: Extensive test on the lattice constant, bulk modulus, and cohesive energy of solids. The Journal of Chemical Physics 2016, 144 (20), 204120.

25. Lejaeghere, K.; Speybroeck, V. V.; Oost, G. V.; Cottenier, S., Error Estimates for Solid-State Density-Functional Theory Predictions: An Overview by Means of the Ground-State Elemental Crystals. Critical Reviews in Solid State and Materials Sciences 2014, 39 (1), 1-24.

26. Zhang, G.-X.; Reilly, A. M.; Tkatchenko, A.; Scheffler, M., Performance of various density-functional approximations for cohesive properties of 64 bulk solids. New Journal of Physics 2018, 20, 063020.

27. Zhang, Y.; Furness, J. W.; Xiao, B.; Sun, J., Subtlety of TiO2 phase stability: Reliability of the density functional theory predictions and persistence of the self-interaction error. J. Chem. Phys. 2019, $150,014105$.

28. Chen, L.; Lau, J. A.; Schwarzer, D.; Meyer, J.; Verma, V. B.; Wodtke, A. M., The Sommerfeld ground-wave limit for a molecule adsorbed at a surface. Science 2019, 363 (6423), 158-161. 
Table S1. The k-grid setting used for different methods. The (semi)-local DFAs include BLYP, PBE, M06-L, and SCAN, while hybrid ones include B3LYP, PBE0, M06, and SCAN0. For XYG3 and XYGJ-OS, the complete k-mesh extrapolation from $6 \times 6 \times 6$ and $8 \times 8 \times 8$, denoted as CKM $(6,8)$, is adopted with $\alpha=3.0$ (see equation S2).

\begin{tabular}{|c|c|c|c|c|c|c|c|}
\hline & \multirow[b]{2}{*}{ Structure } & \multicolumn{2}{|c|}{ (semi)-local DFAs } & \multicolumn{2}{|c|}{ Hybrid DFAs } & \multicolumn{2}{|c|}{ XYG3 and XYGJ-OS } \\
\hline & & Type & k-points & Type & k-points & Type & k-points \\
\hline $\mathrm{C}$ & diamond & MP & $6 \times 6 \times 6$ & GC & 10x10x10 & GC & $\operatorname{CKM}(6,8)$ \\
\hline $\mathrm{Si}$ & diamond & MP & $6 \times 6 \times 6$ & GC & $10 \times 10 \times 10$ & $\mathrm{GC}$ & $\operatorname{CKM}(6,8)$ \\
\hline $\mathrm{LiH}$ & rocksalt & MP & $6 \times 6 \times 6$ & GC & $8 \times 8 \times 8$ & $\mathrm{GC}$ & $\operatorname{CKM}(6,8)$ \\
\hline $\mathrm{LiF}$ & rocksalt & MP & $6 \times 6 \times 6$ & GC & $6 \times 6 \times 6$ & $\mathrm{GC}$ & $\operatorname{CKM}(6,8)$ \\
\hline $\mathrm{LiCl}$ & rocksalt & MP & $6 \times 6 \times 6$ & GC & $6 \times 6 \times 6$ & GC & $\operatorname{CKM}(6,8)$ \\
\hline $\mathrm{NaF}$ & rocksalt & MP & $6 \times 6 \times 6$ & GC & $6 \times 6 \times 6$ & $\mathrm{GC}$ & $\operatorname{CKM}(6,8)$ \\
\hline $\mathrm{NaCl}$ & rocksalt & MP & $6 \times 6 \times 6$ & GC & $6 \times 6 \times 6$ & $\mathrm{GC}$ & $\operatorname{CKM}(6,8)$ \\
\hline $\mathrm{MgO}$ & rocksalt & MP & $6 \times 6 \times 6$ & GC & $6 \times 6 \times 6$ & GC & $\operatorname{CKM}(6,8)$ \\
\hline $\mathrm{MgS}$ & rocksalt & MP & $6 \times 6 \times 6$ & GC & $8 \times 8 \times 8$ & $\mathrm{GC}$ & $\operatorname{CKM}(6,8)$ \\
\hline $\mathrm{BeS}$ & zincblende & MP & $6 \times 6 \times 6$ & GC & $8 \times 8 \times 8$ & $\mathrm{GC}$ & $\operatorname{CKM}(6,8)$ \\
\hline $\mathrm{BN}$ & zincblende & MP & $6 \times 6 \times 6$ & GC & $8 \times 8 \times 8$ & $\mathrm{GC}$ & $\operatorname{CKM}(6,8)$ \\
\hline $\mathrm{BP}$ & zincblende & MP & $6 \times 6 \times 6$ & GC & 10x10x10 & GC & $\operatorname{CKM}(6,8)$ \\
\hline $\mathrm{SiC}$ & zincblende & MP & $6 \times 6 \times 6$ & GC & $10 \times 10 \times 10$ & $\mathrm{GC}$ & $\operatorname{CKM}(6,8)$ \\
\hline AlP & zincblende & MP & $6 \times 6 \times 6$ & GC & $8 \times 8 \times 8$ & $\mathrm{GC}$ & $\operatorname{CKM}(6,8)$ \\
\hline $\mathrm{Ne}$ & fcc & MP & $4 \times 4 \times 4$ & GC & $6 \times 6 \times 6$ & GC & $\operatorname{CKM}(6,8)$ \\
\hline $\mathrm{Ar}$ & fcc & MP & $4 \times 4 \times 4$ & GC & $6 \times 6 \times 6$ & GC & $\operatorname{CKM}(6,8)$ \\
\hline $\mathrm{TiO} 2$ & rutile & $\mathrm{GC}$ & $6 \times 6 \times 8$ & GC & $6 \times 6 \times 8$ & $\mathrm{GC}$ & $4 \times 4 \times 6$ \\
\hline $\mathrm{TiO} 2$ & anatase & GC & $6 \times 6 \times 6$ & GC & $6 \times 6 \times 6$ & GC & $4 \times 4 \times 4$ \\
\hline
\end{tabular}


Table S2. Lattice constants (in A) of 14 cubic crystals together with 2 rare-gas crystals calculated by using varying DFAs obtained in this work as well as the corresponding experimental reference data (Ref.) ${ }^{\mathrm{a}}$ and the values of Møllet-Plesset second order perturbation theory (MP2) and random phase approximation (RPA) from Reference ${ }^{17}$.

\begin{tabular}{|c|c|c|c|c|c|c|c|c|}
\hline & Structure & Ref. $^{\text {a }}$ & $\mathrm{MP} 2^{\mathrm{b}}$ & $\mathrm{RPA}^{\mathrm{b}}$ & XYG3 & XYGJ-OS & BLYP & B3LYP \\
\hline $\mathrm{C}$ & diamond & 3.553 & 3.541 & 3.565 & 3.546 & 3.549 & 3.598 & 3.568 \\
\hline $\mathrm{Si}$ & diamond & 5.421 & 5.404 & 5.455 & 5.424 & 5.431 & 5.534 & 5.487 \\
\hline $\mathrm{LiH}$ & rocksalt & 3.979 & 3.994 & 4.082 & 3.949 & 3.967 & 4.027 & 4.002 \\
\hline $\mathrm{LiF}$ & rocksalt & 3.972 & 4.018 & 3.986 & 3.958 & 3.958 & 4.084 & 4.024 \\
\hline $\mathrm{LiCl}$ & rocksalt & 5.070 & 5.035 & 5.073 & 5.052 & 5.06 & 5.236 & 5.182 \\
\hline $\mathrm{NaF}$ & rocksalt & 4.582 & -- & -- & 4.529 & 4.536 & 4.713 & 4.644 \\
\hline $\mathrm{NaCl}$ & rocksalt & 5.569 & -- & -- & 5.591 & 5.558 & 5.766 & 5.700 \\
\hline $\mathrm{MgO}$ & rocksalt & 4.189 & 4.240 & 4.235 & 4.202 & 4.195 & 4.277 & 4.226 \\
\hline $\mathrm{MgS}$ & rocksalt & $5.182^{\mathrm{c}}$ & 5.182 & 5.213 & 5.181 & 5.183 & 5.295 & 5.249 \\
\hline $\mathrm{BeS}$ & zincblende & $4.849^{c}$ & 4.820 & 4.860 & 4.836 & 4.846 & 4.934 & 4.902 \\
\hline $\mathrm{BN}$ & zincblende & 3.592 & 3.588 & 3.605 & 3.593 & 3.594 & 3.648 & 3.618 \\
\hline $\mathrm{BP}$ & zincblende & 4.525 & 4.498 & 4.544 & 4.516 & 4.524 & 4.598 & 4.564 \\
\hline $\mathrm{SiC}$ & zincblende & 4.346 & 4.350 & 4.369 & 4.352 & 4.352 & 4.417 & 4.380 \\
\hline AlP & zincblende & 5.451 & 5.450 & 5.487 & 5.458 & 5.464 & 5.566 & 5.521 \\
\hline \multicolumn{2}{|c|}{ MAE (in pm) } & & 2.009 & 2.886 & 1.387 & 0.993 & 10.095 & 5.609 \\
\hline $\mathrm{Ne}$ & fcc & 4.314 & 4.499 & 5.611 & 4.256 & 4.266 & $--d$ & $--d$ \\
\hline $\mathrm{Ar}$ & fcc & 5.284 & 5.150 & 5.414 & 5.335 & 5.358 & $-{ }^{d}$ & $--^{d}$ \\
\hline
\end{tabular}

\begin{tabular}{ccccccccc}
\hline & Structure & Ref. & PBE & PBE0 & M06-L & M06 & SCAN & SCAN0 \\
\hline $\mathrm{C}$ & diamond & 3.553 & 3.572 & 3.545 & 3.549 & 3.544 & 3.552 & 3.535 \\
$\mathrm{Si}$ & diamond & 5.421 & 5.470 & 5.439 & 5.415 & 5.442 & 5.431 & 5.416 \\
$\mathrm{LiH}$ & rocksalt & 3.979 & 4.007 & 3.991 & 4.035 & 4.140 & 3.995 & 3.985 \\
$\mathrm{LiF}$ & rocksalt & 3.972 & 4.066 & 4.006 & 3.992 & 4.056 & 3.974 & 3.947 \\
$\mathrm{LiCl}$ & rocksalt & 5.070 & 5.152 & 5.113 & 5.166 & 5.244 & 5.107 & 5.088 \\
$\mathrm{NaF}$ & rocksalt & 4.582 & 4.703 & 4.634 & 4.604 & 4.638 & 4.570 & 4.544 \\
$\mathrm{NaCl}$ & rocksalt & 5.569 & 5.699 & 5.650 & 5.636 & 5.707 & 5.578 & 5.565 \\
$\mathrm{MgO}$ & rocksalt & 4.189 & 4.256 & 4.203 & 4.191 & 4.215 & 4.192 & 4.165 \\
$\mathrm{MgS}$ & rocksalt & 5.182 & 5.231 & 5.197 & 5.185 & 5.235 & 5.195 & 5.178 \\
$\mathrm{BeS}$ & zincblende & 4.849 & 4.876 & 4.857 & 4.874 & 4.910 & 4.866 & 4.852 \\
$\mathrm{BN}$ & zincblende & 3.592 & 3.625 & 3.597 & 3.599 & 3.600 & 3.606 & 3.587 \\
$\mathrm{BP}$ & zincblende & 4.525 & 4.548 & 4.525 & 4.512 & 4.532 & 4.527 & 4.511 \\
$\mathrm{SiC}$ & zincblende & 4.346 & 4.381 & 4.352 & 4.324 & 4.336 & 4.354 & 4.335 \\
$\mathrm{AlP}$ & zincblende & 5.451 & 5.508 & 5.476 & 5.446 & 5.483 & 5.470 & 5.454 \\
\hline \multicolumn{2}{c}{$\mathrm{MAE}$ (in pm) } & & 5.815 & 2.286 & 2.488 & 6.007 & 1.166 & 1.271 \\
\hline $\mathrm{Ne}$ & fcc & 4.314 & 4.604 & 4.594 & 4.301 & 4.877 & 4.076 & 4.108 \\
$\mathrm{Ar}$ & fcc & 5.284 & 5.944 & 5.956 & 5.259 & 5.289 & 5.329 & 5.365 \\
\hline
\end{tabular}

a): Experimental reference values are corrected from thermal and zero-point vibrational effects. Most of them are collected from Refereces ${ }^{24}$ and ${ }^{25}$. b) The MP2 and RPA results are collected 
from Reference ${ }^{17}$. c): Experimental reference values of MgS is collected from Reference ${ }^{26}$. The thermal and zero-point vibrational effects are excluded at the PBE level of theory. For BeS, we don't find reliable experimental reference number. In consequence, we take the average number of XYG3, XYGJ-OS, SCAN, and SCANO as the reference, considering the excellent overall performance of these four DFAs to the problem. d): BLYP and B3LYP predict a completely repulsive dissociation curves for both $\mathrm{Ne}$ and $\mathrm{Ar}$ crystals. 
Table S3. Bulk modulus (in GPa) of 14 cubic crystals together with 2 rare-gas crystals calculated by using varying DFAs obtained in this work as well as the corresponding experimental reference data (Ref.) ${ }^{\mathrm{a}}$ and the values of Møllet-Plesset second order perturbation theory (MP2) and random phase approximation (RPA) from Reference ${ }^{17}$.

\begin{tabular}{|c|c|c|c|c|c|c|c|c|}
\hline & Structure & Ref. $^{a}$ & $\mathrm{MP} 2^{\mathrm{b}}$ & $\mathrm{RPA}^{\mathrm{b}}$ & XYG3 & XYGJ-OS & BLYP & B3LYP \\
\hline $\mathrm{C}$ & diamond & 455.0 & 454.8 & 437.9 & 459.4 & 457.1 & 406.6 & 443.4 \\
\hline $\mathrm{Si}$ & diamond & 101.0 & 100.2 & 95.7 & 96.1 & 97.7 & 78.8 & 89.7 \\
\hline $\mathrm{LiH}$ & rocksalt & 40.1 & 38.5 & 34.7 & 39.0 & 37 & 34.8 & 36.4 \\
\hline $\mathrm{LiF}$ & rocksalt & 76.0 & 78.3 & 82.0 & 80.5 & 72 & 66.4 & 73.7 \\
\hline $\mathrm{LiCl}$ & rocksalt & 39.0 & 38.7 & 38.0 & 38.5 & 37.9 & 28.9 & 31.5 \\
\hline $\mathrm{NaF}$ & rocksalt & 53.1 & -- & -- & 49.3 & 51.6 & 44.9 & 50.1 \\
\hline $\mathrm{NaCl}$ & rocksalt & 27.6 & -- & -- & 28.6 & 28.9 & 21.9 & 24.0 \\
\hline $\mathrm{MgO}$ & rocksalt & 170.0 & 156.7 & 159.4 & 165.6 & 167.5 & 145.8 & 161.8 \\
\hline $\mathrm{MgS}$ & rocksalt & 80.0 & 84.0 & 81.3 & 83.8 & 83.5 & 68.3 & 74.7 \\
\hline $\mathrm{BeS}$ & zincblende & 100.8 & 104.5 & 100.9 & 102.9 & 101.5 & 86.6 & 93.2 \\
\hline $\mathrm{BN}$ & zincblende & 410.0 & 368.4 & 370.1 & 401.8 & 400.5 & 354.9 & 385.5 \\
\hline BP & zincblende & 168.0 & 178.1 & 167.7 & 175.0 & 173.5 & 148.9 & 163.1 \\
\hline $\mathrm{SiC}$ & zincblende & 229.0 & 230.1 & 222.0 & 226.2 & 226.9 & 197.2 & 217.0 \\
\hline AlP & zincblende & 87.0 & 95.5 & 90.4 & 92.5 & 92.6 & 75.0 & 84.0 \\
\hline \multicolumn{2}{|c|}{ MAE (in GPa) } & & 7.3 & 8.1 & 3.8 & 3.3 & 19.8 & 7.8 \\
\hline $\mathrm{Ne}$ & fcc & 2.8 & 1.0 & 0.2 & 2.3 & 2.3 & $--d$ & $--d$ \\
\hline \multirow[t]{2}{*}{$\mathrm{Ar}$} & fcc & 3.3 & 3.7 & 1.7 & 2.5 & 2.4 & $--d$ & $-{ }^{d}$ \\
\hline & Structure & Ref. & PBE & PBE0 & M06-L & M06 & SCAN & SCAN0 \\
\hline $\mathrm{C}$ & diamond & 455.0 & 432.9 & 471.2 & 454.2 & 461.2 & 458.0 & 484.1 \\
\hline $\mathrm{Si}$ & diamond & 101.0 & 88.7 & 99.4 & 100.0 & 97.0 & 99.5 & 104.7 \\
\hline $\mathrm{LiH}$ & rocksalt & 40.1 & 36.2 & 37.3 & 28.0 & 27.1 & 36.6 & 37.4 \\
\hline $\mathrm{LiF}$ & rocksalt & 76.0 & 67.0 & 74.6 & 68.5 & 66.7 & 79.9 & 83.7 \\
\hline $\mathrm{LiCl}$ & rocksalt & 39.0 & 31.8 & 33.9 & 31.5 & 31.2 & 36.3 & 36.7 \\
\hline $\mathrm{NaF}$ & rocksalt & 53.1 & 44.9 & 50.0 & 46.8 & 48.0 & 56.9 & 59.0 \\
\hline $\mathrm{NaCl}$ & rocksalt & 27.6 & 23.6 & 25.2 & 23.4 & 24.2 & 29.0 & 29.3 \\
\hline $\mathrm{MgO}$ & rocksalt & 170.0 & 149.0 & 167.0 & 165.7 & 154.2 & 169.5 & 180.7 \\
\hline $\mathrm{MgS}$ & rocksalt & 80.0 & 74.1 & 80.3 & 78.4 & 71.5 & 80.3 & 83.3 \\
\hline $\mathrm{BeS}$ & zincblende & 100.8 & 93.0 & 99.0 & 95.0 & 91.1 & 97.4 & 100.9 \\
\hline $\mathrm{BN}$ & zincblende & 410.0 & 371.8 & 404.1 & 391.9 & 394.3 & 392.1 & 414.3 \\
\hline $\mathrm{BP}$ & zincblende & 168.0 & 162.0 & 175.4 & 172.7 & 167.1 & 173.4 & 181.3 \\
\hline $\mathrm{SiC}$ & zincblende & 229.0 & 212.1 & 231.5 & 228.5 & 228.4 & 226.3 & 239.9 \\
\hline AlP & zincblende & 87.0 & 82.5 & 91.3 & 91.7 & 87.0 & 90.7 & 95.5 \\
\hline \multicolumn{2}{|c|}{ MAE (in GPa) } & & 11.9 & 4.1 & 5.7 & 7.1 & 3.8 & 7.4 \\
\hline $\mathrm{Ne}$ & fcc & 2.8 & 1.2 & 1.0 & 3.7 & 1.1 & 4.5 & 3.7 \\
\hline $\mathrm{Ar}$ & $\mathrm{fcc}$ & 3.3 & 0.7 & 0.6 & 2.8 & 0.8 & 2.8 & 2.4 \\
\hline
\end{tabular}

a): Experimental reference values are corrected from thermal and zero-point vibrational effects. Most of them are collected from Refereces ${ }^{24}$ and ${ }^{25}$. b): The MP2 and RPA results are collected 
from Reference ${ }^{17}$. c): Experimental reference values of MgS is collected from Reference ${ }^{26}$. The thermal and zero-point vibrational effects are excluded at the PBE level of theory. For BeS, we don't find reliable experimental reference number. In consequence, we take the average number of XYG3, XYGJ-OS, SCAN, and SCANO as the reference, considering the excellent overall performance of these four DFAs to the problem. d): BLYP and B3LYP predict a completely repulsive dissociation curves for both $\mathrm{Ne}$ and $\mathrm{Ar}$ crystals. 
Table S4. Cohesive energies per atom (in eV) of 14 cubic crystals together with 2 rare-gas crystals calculated by using varying DFAs obtained in this work as well as the corresponding experimental reference data (Ref.) ${ }^{\text {a }}$ and the values of Møllet-Plesset second order perturbation theory (MP2) and random phase approximation (RPA) from Reference ${ }^{17}$.

\begin{tabular}{|c|c|c|c|c|c|c|c|c|}
\hline & Structure & Ref. $^{a}$ & $\mathrm{MP} 2^{\mathrm{b}}$ & $\mathrm{RPA}^{\mathrm{b}}$ & XYG3 & XYGJ-OS & BLYP & B3LYP \\
\hline $\mathrm{C}$ & diamond & 7.550 & 8.083 & 7.068 & 7.538 & 7.539 & 7.012 & 7.111 \\
\hline $\mathrm{Si}$ & diamond & 4.680 & 5.214 & 4.412 & 4.885 & 4.763 & 3.967 & 4.109 \\
\hline $\mathrm{LiH}$ & rocksalt & 2.490 & 2.382 & 2.256 & 2.510 & 2.508 & 2.323 & 2.388 \\
\hline $\mathrm{LiF}$ & rocksalt & 4.460 & 4.544 & 4.076 & 4.475 & 4.443 & 4.286 & 4.299 \\
\hline $\mathrm{LiCl}$ & rocksalt & 3.590 & 3.698 & 3.332 & 3.597 & 3.558 & 3.218 & 3.304 \\
\hline $\mathrm{NaF}$ & rocksalt & 3.970 & -- & -- & 3.985 & 3.968 & 3.765 & 3.776 \\
\hline $\mathrm{NaCl}$ & rocksalt & 3.340 & -- & -- & 3.334 & 3.31 & 2.950 & 3.044 \\
\hline $\mathrm{MgO}$ & rocksalt & 5.200 & 5.532 & 4.940 & 5.186 & 5.164 & 4.725 & 4.792 \\
\hline $\mathrm{MgS}$ & rocksalt & 4.040 & 4.389 & 3.816 & 3.994 & 3.929 & 3.269 & 3.425 \\
\hline $\mathrm{BeS}$ & zincblende & 4.556 & 4.902 & 4.253 & 4.562 & 4.479 & 4.033 & 4.153 \\
\hline $\mathrm{BN}$ & zincblende & 6.760 & 7.247 & 6.506 & 6.856 & 6.848 & 6.452 & 6.529 \\
\hline BP & zincblende & 5.140 & 5.773 & 4.993 & 5.407 & 5.324 & 4.693 & 4.813 \\
\hline $\mathrm{SiC}$ & zincblende & 6.480 & 7.010 & 6.073 & 6.583 & 6.516 & 5.781 & 5.934 \\
\hline AlP & zincblende & 4.320 & 4.671 & 4.103 & 4.380 & 4.308 & 3.624 & 3.765 \\
\hline \multicolumn{2}{|c|}{ MAE (in kcal/mol) } & & 8.44 & 6.61 & 1.44 & 1.21 & 10.67 & 8.45 \\
\hline $\mathrm{Ne}$ & fcc & 0.026 & 0.014 & 0.001 & 0.016 & 0.021 & $-d^{d}$ & $--d$ \\
\hline \multirow[t]{2}{*}{$\mathrm{Ar}$} & fcc & 0.083 & 0.087 & 0.042 & 0.047 & 0.051 & $-{ }^{d}$ & $--^{d}$ \\
\hline & Structure & Ref. & PBE & PBE0 & M06-L & M06 & SCAN & SCAN0 \\
\hline $\mathrm{C}$ & diamond & 7.550 & 7.737 & 7.629 & 7.426 & 7.380 & 7.512 & 7.420 \\
\hline $\mathrm{Si}$ & diamond & 4.680 & 4.585 & 4.594 & 4.738 & 4.566 & 4.737 & 4.663 \\
\hline $\mathrm{LiH}$ & rocksalt & 2.490 & 2.356 & 2.366 & 2.597 & 2.478 & 2.443 & 2.448 \\
\hline $\mathrm{LiF}$ & rocksalt & 4.460 & 4.328 & 4.236 & 4.511 & 4.448 & 4.405 & 4.312 \\
\hline $\mathrm{LiCl}$ & rocksalt & 3.590 & 3.376 & 3.387 & 3.792 & 3.616 & 3.536 & 3.506 \\
\hline $\mathrm{NaF}$ & rocksalt & 3.970 & 3.833 & 3.736 & 4.172 & 4.069 & 3.957 & 3.852 \\
\hline $\mathrm{NaCl}$ & rocksalt & 3.340 & 3.113 & 3.136 & 3.682 & 3.474 & 3.335 & 3.300 \\
\hline $\mathrm{MgO}$ & rocksalt & 5.200 & 5.003 & 4.933 & 5.302 & 5.033 & 5.283 & 5.133 \\
\hline $\mathrm{MgS}$ & rocksalt & 4.040 & 3.741 & 3.799 & 4.161 & 3.834 & 4.046 & 3.990 \\
\hline $\mathrm{BeS}$ & zincblende & 4.556 & 4.478 & 4.457 & 4.575 & 4.426 & 4.605 & 4.548 \\
\hline $\mathrm{BN}$ & zincblende & 6.760 & 6.952 & 6.849 & 6.733 & 6.711 & 6.847 & 6.727 \\
\hline BP & zincblende & 5.140 & 5.315 & 5.275 & 5.207 & 5.086 & 5.340 & 5.260 \\
\hline $\mathrm{SiC}$ & zincblende & 6.480 & 6.433 & 6.414 & 6.484 & 6.376 & 6.487 & 6.400 \\
\hline AlP & zincblende & 4.320 & 4.118 & 4.140 & 4.265 & 4.154 & 4.289 & 4.226 \\
\hline \multicolumn{2}{|c|}{ MAE (in GPa) } & & 3.82 & 3.67 & 2.44 & 2.38 & 1.21 & 1.70 \\
\hline $\mathrm{Ne}$ & $\mathrm{fcc}$ & 0.026 & 0.019 & 0.014 & 0.066 & 0.031 & 0.048 & 0.039 \\
\hline $\mathrm{Ar}$ & $\mathrm{fcc}$ & 0.083 & 0.022 & 0.016 & 0.105 & 0.047 & 0.056 & 0.047 \\
\hline
\end{tabular}

a): Experimental reference values are corrected from thermal and zero-point vibrational effects. Most of them are collected from Refereces ${ }^{24}$ and ${ }^{25}$. b): The MP2 and RPA results are collected 
from Reference ${ }^{17}$. c): Experimental reference values of MgS is collected from Reference ${ }^{26}$. The thermal and zero-point vibrational effects are excluded at the PBE level of theory. For BeS, we don't find reliable experimental reference number. In consequence, we take the average number of XYG3, XYGJ-OS, SCAN, and SCANO as the reference, considering the excellent overall performance of these four DFAs to the problem. d): BLYP and B3LYP predict a completely repulsive dissociation curves for both $\mathrm{Ne}$ and $\mathrm{Ar}$ crystals. 
Table S5. Equilibrium volume of the primitive cell $V_{0}$, the bulk modulus $B_{0}$, and the energy difference between rutile and anatase $\Delta E_{r-a}$.

\begin{tabular}{|c|c|c|c|c|c|}
\hline \multirow[b]{2}{*}{ Methods } & \multicolumn{2}{|c|}{$V_{0}\left[\AA^{3}\right]$} & \multicolumn{2}{|c|}{$B_{0}[\mathrm{GPa}]$} & \multirow[b]{2}{*}{$\Delta E_{r-a}[\mathrm{~kJ} / \mathrm{mol}]$} \\
\hline & Rutile & Anatase & Rutile & Anatase & \\
\hline PBE & 31.92 & 34.98 & 199.7 & 172.6 & 9.26 \\
\hline SCAN & 31.01 & 34.00 & 235.0 & 190.9 & 2.81 \\
\hline M06-L & 31.50 & $--^{\mathrm{a}}$ & 225.3 & $--^{\mathrm{a}}$ & $--^{\mathrm{a}}$ \\
\hline B3LYP@PBE & 31.64 & 34.74 & 217.0 & 192.8 & 10.54 \\
\hline SCAN0@PBE & 30.37 & 33.42 & 259.5 & 215.9 & 2.19 \\
\hline SCAN0@SCAN & 30.37 & 33.36 & 261.3 & 212.7 & 2.80 \\
\hline M06@PBE & 31.13 & 33.96 & 235.4 & 203.2 & 4.90 \\
\hline XYG3@PBE & 31.33 & 34.01 & 204.9 & 166.7 & -6.73 \\
\hline XYG3@SCAN & 31.24 & 34.01 & 207.2 & 168.7 & -7.19 \\
\hline XYGJ-OS@PBE & 31.02 & 33.84 & 215.5 & 180.3 & -2.80 \\
\hline XYGJ-OS@SCAN & 31.02 & 33.86 & 216.3 & 179.2 & -3.04 \\
\hline Expt. $^{b}$ & 31.08 & 33.98 & 216.0 & 178.0 & -0.76 \\
\hline
\end{tabular}

a): The self-consistent convergence of M06-L calculations fails at some points of Anatase TiO2.

b): The experimental volume and bulk modulus are taken from References ${ }^{18}$ and ${ }^{27}$. The ZPE contribution from the PBEsol calculations has been excluded in the experimental energy difference. ${ }^{18}$ 
Table S6. Bond dissociation energies calculated by varying DFAs, as well as the experimental references

\begin{tabular}{|c|c|c|c|c|c|}
\hline Methods & Ref. & XYG3 & XYGJ-OS & BLYP & B3LYP \\
\hline $\mathrm{H}-\mathrm{OH} \rightarrow \mathrm{OH}+\mathrm{H}$ & 119.30 & 117.95 & 118.68 & 116.05 & 115.95 \\
\hline $\mathrm{H}-\mathrm{OCl}->\mathrm{OCl}+\mathrm{H}$ & 94.09 & 93.55 & 96.36 & 88.82 & 91.73 \\
\hline $\mathrm{CH} 3 \mathrm{O}-\mathrm{H}-->\mathrm{CH} 3 \mathrm{O}+\mathrm{H}$ & 104.20 & 103.97 & 105.15 & 98.08 & 99.87 \\
\hline $\mathrm{C} 2 \mathrm{H} 5 \mathrm{O}-\mathrm{H}->\mathrm{C} 2 \mathrm{H} 5 \mathrm{O}+\mathrm{H}$ & 104.61 & 104.09 & 105.29 & 97.41 & 99.55 \\
\hline $\mathrm{H}-\mathrm{SH}-->\mathrm{HS}+\mathrm{H}$ & 91.18 & 91.06 & 91.78 & 88.56 & 89.48 \\
\hline $\mathrm{CH} 3 \mathrm{~S}-\mathrm{H}-->\mathrm{CH} 3 \mathrm{~S}+\mathrm{H}$ & 87.40 & 86.45 & 87.30 & 82.88 & 84.23 \\
\hline $\mathrm{H}-\mathrm{NH} 2->\mathrm{NH} 2+\mathrm{H}$ & 108.17 & 107.20 & 107.91 & 104.10 & 105.05 \\
\hline $\mathrm{H}-\mathrm{NH}-\rightarrow \mathrm{NH}+\mathrm{H}$ & 92.20 & 93.82 & 94.80 & 94.08 & 94.02 \\
\hline $\mathrm{H}-\mathrm{PH} 2 \rightarrow \mathrm{PH} 2+\mathrm{H}$ & 83.90 & 82.56 & 83.35 & 79.92 & 81.10 \\
\hline $\mathrm{CH} 3-\mathrm{H}->\mathrm{CH} 3+\mathrm{H}$ & 105.00 & 104.81 & 105.67 & 102.28 & 103.37 \\
\hline $\mathrm{CH} 2-\mathrm{H}->\mathrm{CH} 2(3 \mathrm{~B} 1)+\mathrm{H}$ & 110.80 & 112.02 & 112.72 & 110.61 & 111.44 \\
\hline $\mathrm{CH} 2(3 \mathrm{~B} 1)-->\mathrm{CH}+\mathrm{H}$ & 100.90 & 101.98 & 100.37 & 99.32 & 101.54 \\
\hline $\mathrm{C} 2 \mathrm{H} 5-\mathrm{H}-\rightarrow \mathrm{C} 2 \mathrm{H} 5+\mathrm{H}$ & 101.08 & 101.09 & 102.02 & 97.13 & 98.67 \\
\hline$(\mathrm{CH} 3)_{2} \mathrm{CH}-\mathrm{H}->(\mathrm{CH} 3) 2 \mathrm{CH}+\mathrm{H}$ & 98.60 & 98.35 & 99.32 & 93.04 & 94.94 \\
\hline (CH3)3C-H --> (CH3)3C $+\mathrm{H}$ & 96.47 & 96.54 & 97.54 & 90.02 & 92.23 \\
\hline $\mathrm{CH} 2=\mathrm{CH}-\mathrm{H}->\mathrm{CH} 2 \mathrm{CH}+\mathrm{H}$ & 111.16 & 110.88 & 111.81 & 106.54 & 108.52 \\
\hline $\mathrm{HCC}-\mathrm{H} \rightarrow \mathrm{CCH}+\mathrm{H}$ & 133.01 & 134.89 & 135.29 & 132.22 & 133.88 \\
\hline $\mathrm{C} 6 \mathrm{H} 5-\mathrm{H}->\mathrm{C} 6 \mathrm{H} 5+\mathrm{H}$ & 113.56 & 113.11 & 114.08 & 108.40 & 110.77 \\
\hline $\mathrm{CH} 3 \mathrm{OH}-->\mathrm{CH} 2 \mathrm{OH}+\mathrm{H}$ & 96.02 & 95.97 & 97.09 & 91.57 & 93.57 \\
\hline $\mathrm{H}-\mathrm{CN} \rightarrow \mathrm{CN}+\mathrm{H}$ & 125.50 & 125.30 & 126.51 & 123.57 & 128.43 \\
\hline $\mathrm{H}-\mathrm{CF} 3-\rightarrow \mathrm{CF} 3+\mathrm{H}$ & 107.40 & 106.45 & 107.66 & 101.51 & 104.01 \\
\hline $\mathrm{H} 2 \mathrm{CO}-->\mathrm{HCO}+\mathrm{H}$ & 88.06 & 87.26 & 88.94 & 84.22 & 86.56 \\
\hline $\mathrm{H}-\mathrm{CO}->\mathrm{CO}+\mathrm{H}$ & 15.68 & 17.34 & 16.76 & 21.52 & 21.42 \\
\hline $\mathrm{CH} 3 \mathrm{CHO}->\mathrm{CH} 3 \mathrm{CO}+\mathrm{H}$ & 89.40 & 88.47 & 90.08 & 84.74 & 87.20 \\
\hline $\mathrm{CH} 3-\mathrm{O}-\rightarrow \mathrm{CH} 3+\mathrm{O}$ & 90.46 & 88.30 & 90.54 & 94.88 & 91.07 \\
\hline $\mathrm{C} 2 \mathrm{H} 5-\mathrm{O}->\mathrm{C} 2 \mathrm{H} 5+\mathrm{O}$ & 92.16 & 90.05 & 92.17 & 95.42 & 91.75 \\
\hline $\mathrm{CH} 3-\mathrm{S}->\mathrm{CH} 3+\mathrm{S}$ & 71.40 & 71.16 & 72.85 & 69.75 & 69.43 \\
\hline $\mathrm{CF} 3-\mathrm{F}-\mathrm{-}>\mathrm{CF} 3+\mathrm{F}$ & 130.72 & 127.02 & 129.77 & 123.09 & 123.28 \\
\hline $\mathrm{CH} 2=\mathrm{CH}-\mathrm{F}->\mathrm{CH} 2 \mathrm{CH}+\mathrm{F}$ & 123.78 & 121.96 & 124.34 & 122.84 & 120.77 \\
\hline $\mathrm{C} 6 \mathrm{H} 5-\mathrm{F}->\mathrm{C} 6 \mathrm{H} 5+\mathrm{F}$ & 127.88 & 124.24 & 126.76 & 124.84 & 123.25 \\
\hline $\mathrm{CH} 3 \mathrm{COF}-->\mathrm{CH} 3 \mathrm{CO}+\mathrm{F}$ & 122.28 & 118.93 & 121.34 & 118.65 & 117.36 \\
\hline $\mathrm{CH} 3 \mathrm{Cl}->>\mathrm{CH} 3+\mathrm{Cl}$ & 83.55 & 81.76 & 83.35 & 78.22 & 78.20 \\
\hline $\mathrm{CH} 3 \mathrm{CH} 2-\mathrm{Cl}->>\mathrm{CH} 3 \mathrm{CH} 2+\mathrm{Cl}$ & 84.69 & 82.56 & 83.98 & 77.06 & 77.44 \\
\hline$(\mathrm{CH} 3) 3 \mathrm{C}-\mathrm{Cl}-->(\mathrm{CH} 3) 3 \mathrm{C}+\mathrm{Cl}$ & 84.79 & 82.71 & 83.95 & 73.07 & 74.20 \\
\hline $\mathrm{CF} 3-\mathrm{Cl}->\mathrm{CF} 3+\mathrm{Cl}$ & 87.19 & 83.68 & 85.51 & 78.06 & 78.93 \\
\hline $\mathrm{CH} 2=\mathrm{CH}-\mathrm{Cl}-->\mathrm{CH} 2 \mathrm{CH}+\mathrm{Cl}$ & 91.69 & 92.97 & 94.26 & 88.16 & 88.54 \\
\hline $\mathrm{C} 6 \mathrm{H} 5-\mathrm{Cl}->\mathrm{C} 6 \mathrm{H} 5+\mathrm{Cl}$ & 97.79 & 95.26 & 96.46 & 89.07 & 89.90 \\
\hline $\mathrm{CH} 3 \mathrm{COCl}->\mathrm{CH} 3 \mathrm{CO}+\mathrm{Cl}$ & 84.59 & 81.85 & 83.16 & 78.44 & 78.32 \\
\hline $\mathrm{HO}-\mathrm{Cl}->>\mathrm{HO}+\mathrm{Cl}$ & 56.19 & 52.45 & 55.18 & 55.97 & 51.34 \\
\hline $\mathrm{Cl}-\mathrm{NO}-\rightarrow \mathrm{NO}+\mathrm{Cl}$ & 38.21 & 36.26 & 38.26 & 45.49 & 36.92 \\
\hline $\mathrm{SiH} 2-\mathrm{H}->\mathrm{SiH} 2+\mathrm{H}$ & 90.40 & 92.53 & 93.04 & 89.68 & 91.24 \\
\hline
\end{tabular}




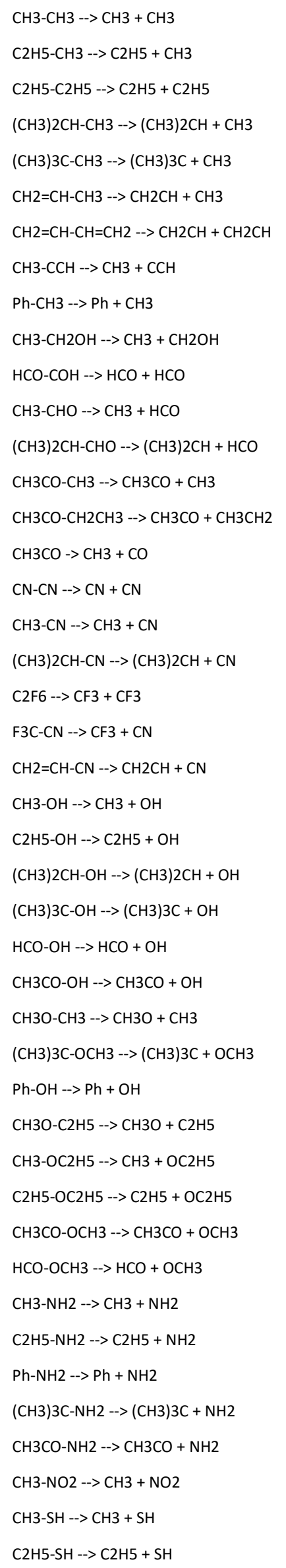

$\begin{array}{ccccc}90.08 & 88.95 & 91.04 & 83.37 & 84.59 \\ 88.90 & 87.66 & 89.69 & 79.79 & 81.55 \\ 87.80 & 86.34 & 88.31 & 76.13 & 78.44 \\ 88.57 & 86.49 & 88.48 & 76.36 & 78.60 \\ 87.50 & 85.22 & 87.15 & 72.75 & 75.47 \\ 101.82 & 100.42 & 102.33 & 92.96 & 95.01 \\ 116.90 & 115.16 & 116.55 & 106.63 & 109.09 \\ 125.90 & 127.36 & 128.66 & 122.61 & 124.35 \\ 104.20 & 102.23 & 104.15 & 93.42 & 95.94 \\ 87.13 & 85.70 & 87.87 & 77.68 & 79.84 \\ 70.70 & 67.97 & 71.16 & 62.09 & 65.30 \\ 84.70 & 82.63 & 85.07 & 77.11 & 79.46 \\ 83.10 & 79.19 & 81.44 & 69.25 & 72.54 \\ 84.53 & 82.16 & 84.66 & 74.59 & 77.40 \\ 83.60 & 81.09 & 83.50 & 70.91 & 74.35 \\ 10.98 & 11.49 & 11.75 & 13.88 & 13.68 \\ 136.50 & 134.90 & 136.60 & 135.51 & 141.93 \\ 121.90 & 120.84 & 122.90 & 117.17 & 122.21 \\ 120.80 & 117.48 & 119.38 & 109.55 & 115.49 \\ 98.70 & 94.12 & 97.08 & 82.36 & 86.64 \\ 112.00 & 109.66 & 112.29 & 104.20 & 109.50 \\ 133.30 & 130.14 & 131.86 & 125.66 & 130.88 \\ 92.40 & 90.46 & 92.74 & 87.72 & 87.09 \\ 94.51 & 92.33 & 94.51 & 87.58 & 87.45 \\ 96.10 & 93.42 & 95.53 & 86.82 & 87.08 \\ 96.40 & 93.88 & 95.98 & 85.39 & 86.08 \\ 109.90 & 107.76 & 109.81 & 104.19 & 104.63 \\ 110.40 & 107.72 & 109.92 & 102.18 & 103.31 \\ 83.10 & 81.86 & 84.31 & 74.13 & 75.46 \\ 84.20 & 82.15 & 84.34 & 67.56 & 70.38 \\ 113.60 & 110.27 & 112.47 & 105.70 & 106.04 \\ 84.70 & 83.77 & 86.08 & 73.86 & 75.71 \\ 83.00 & 82.01 & 84.45 & 73.32 & 75.03 \\ 85.50 & 83.88 & 86.18 & 72.96 & 75.19 \\ 100.10 & 98.20 & 100.46 & 87.04 & 90.20 \\ 99.10 & 98.64 & 100.76 & 89.66 & 92.10 \\ 85.60 & 83.28 & 85.42 & 78.17 & 79.00 \\ 85.30 & 83.80 & 85.84 & 76.69 & 77.95 \\ 105.50 & 103.54 & 105.53 & 96.34 & 98.22 \\ 86.30 & 83.90 & 85.84 & 72.24 & 74.48 \\ 99.70 & 97.04 & 99.15 & 89.95 & 92.52 \\ 60.71 & 56.87 & 61.29 & 51.27 & 54.49 \\ 74.68 & 73.34 & 74.99 & 68.39 & 68.92 \\ 74.18 & 72.43 & 73.96 & 65.21 & 66.19\end{array}$




\begin{tabular}{|c|c|c|c|c|c|}
\hline$(\mathrm{CH} 3) 3 \mathrm{C}-\mathrm{SH}-->(\mathrm{CH} 3) 3 \mathrm{C}+\mathrm{SH}$ & 72.68 & 70.99 & 72.31 & 59.21 & 60.95 \\
\hline CH3S-CH3 --> CH3S + CH3 & 73.70 & 71.73 & 73.33 & 65.03 & 66.05 \\
\hline $\mathrm{HO}-\mathrm{OH}-->\mathrm{OH}+\mathrm{OH}$ & 51.33 & 46.16 & 50.01 & 51.79 & 46.05 \\
\hline $\mathrm{H} 2 \mathrm{~N}-\mathrm{NH} 2-->\mathrm{NH} 2+\mathrm{NH} 2$ & 67.41 & 63.99 & 66.47 & 60.72 & 60.73 \\
\hline CH3O-NO --> CH3O + NO & 41.58 & 40.58 & 42.90 & 40.78 & 36.52 \\
\hline CH3-SiH3 --> CH3 + SiH3 & 89.90 & 88.42 & 89.49 & 80.80 & 82.73 \\
\hline SiH3-SiH3 -> SiH3 + SiH3 & 76.70 & 76.17 & 77.07 & 68.70 & 70.84 \\
\hline $\mathrm{HCCl} 3->>\mathrm{CCl} 3+\mathrm{H}$ & 93.76 & 92.90 & 94.37 & 87.86 & 90.11 \\
\hline HONO $-\rightarrow$ H + NO2 & 78.81 & 74.87 & 77.46 & 72.42 & 75.16 \\
\hline HONO2 --> H + NO3 & 101.20 & 94.96 & 102.21 & 88.46 & 97.90 \\
\hline $\mathrm{n}-\mathrm{C} 3 \mathrm{H} 7 \mathrm{~F}-\rightarrow>\mathrm{n}-\mathrm{C} 3 \mathrm{H} 7+\mathrm{F}$ & 110.08 & 110.66 & 113.05 & 110.80 & 108.74 \\
\hline $\mathrm{ClCN}-->\mathrm{Cl}+\mathrm{CN}$ & 100.89 & 99.20 & 100.65 & 98.49 & 101.13 \\
\hline $\mathrm{CCl} 4->\mathrm{CCl}+\mathrm{Cl}$ & 68.93 & 64.98 & 66.65 & 58.26 & 58.23 \\
\hline i-C3H7Cl --> i-C3H7 + Cl & 85.49 & 82.89 & 84.20 & 75.34 & 76.09 \\
\hline $\mathrm{C} 2 \mathrm{H} 5 \mathrm{OOC} 2 \mathrm{H} 5$--> $2 \mathrm{C} 2 \mathrm{H} 5 \mathrm{O}$ & 38.70 & 34.27 & 37.96 & 28.71 & 26.93 \\
\hline $\mathrm{NO} 3-\rightarrow \mathrm{NO} 2+\mathrm{O}$ & 50.47 & 51.45 & 49.52 & 63.00 & 51.17 \\
\hline HONO --> HO + NO & 49.78 & 47.32 & 50.05 & 53.41 & 47.23 \\
\hline HONO2 --> HO + NO2 & 49.41 & 44.60 & 48.78 & 46.21 & 45.23 \\
\hline $\mathrm{C} 2 \mathrm{H} 5 \mathrm{ONO}-->\mathrm{C} 2 \mathrm{H} 5 \mathrm{O}+\mathrm{NO}$ & 43.78 & 39.78 & 42.49 & 39.47 & 35.63 \\
\hline $\mathrm{C} 2 \mathrm{H} 5 \mathrm{NO} 3$--> C2H5O + NO2 & 41.01 & 37.56 & 41.43 & 32.81 & 33.92 \\
\hline $\mathrm{HSSH}-->2 \mathrm{HS}$ & 64.66 & 62.20 & 63.40 & 59.53 & 58.68 \\
\hline $\mathrm{CH} 3 \mathrm{SSCH}_{3}-->2 \mathrm{CH} 3 \mathrm{~S}$ & 65.40 & 61.76 & 62.50 & 55.55 & 55.42 \\
\hline $\mathrm{n}-\mathrm{C} 3 \mathrm{H} 7 \mathrm{NO3}-->\mathrm{n}-\mathrm{C} 3 \mathrm{H} 7+\mathrm{NO} 3$ & 82.50 & 76.22 & 84.10 & 65.05 & 74.41 \\
\hline i-C3H7NO3 --> i-C3H7 + NO3 & 84.10 & 76.93 & 84.65 & 63.61 & 73.36 \\
\hline $\mathrm{C} 2 \mathrm{H} 5 \mathrm{NO} 3 \rightarrow->\mathrm{C} 2 \mathrm{H} 5+\mathrm{NO} 3$ & 82.70 & 76.17 & 84.08 & 65.23 & 74.50 \\
\hline $\mathrm{n}-\mathrm{C} 5 \mathrm{H} 12->\mathrm{n}-\mathrm{C} 3 \mathrm{H} 7+\mathrm{C} 2 \mathrm{H} 5$ & 87.90 & 86.59 & 88.53 & 76.09 & 78.47 \\
\hline $\mathrm{n}-\mathrm{C} 6 \mathrm{H} 14-->2 \mathrm{n}-\mathrm{C} 3 \mathrm{H} 7$ & 87.70 & 86.88 & 88.78 & 76.13 & 78.58 \\
\hline $\mathrm{C} 2 \mathrm{H} 5 \mathrm{CH}(\mathrm{CH} 3) 2->\mathrm{C} 2 \mathrm{H} 5+\mathrm{CH}(\mathrm{CH} 3) 2$ & 87.30 & 84.45 & 86.36 & 71.59 & 74.44 \\
\hline $\mathrm{C} 2 \mathrm{H} 5 \mathrm{CH}(\mathrm{CH} 3) 2-\rightarrow \mathrm{CH} 3+\mathrm{CH} 2 \mathrm{CH}(\mathrm{CH} 3) 2$ & 88.90 & 87.62 & 89.60 & 79.04 & 80.97 \\
\hline$(\mathrm{CH} 3)_{2} \mathrm{CHC}(\mathrm{CH} 3) 3-->(\mathrm{CH} 3) 2 \mathrm{CH}+\mathrm{C}(\mathrm{CH} 3) 3$ & 82.70 & 78.90 & 80.64 & 60.02 & 64.03 \\
\hline$(\mathrm{CH} 3)_{2} \mathrm{CH}(\mathrm{CH} 2) 2 \mathrm{OH}->(\mathrm{CH} 3) 2 \mathrm{CHCH} 2+\mathrm{CH} 2 \mathrm{OH}$ & 85.12 & 84.14 & 86.18 & 72.88 & 75.80 \\
\hline $\mathrm{F} 3 \mathrm{CCH} 3-->\mathrm{F} 3 \mathrm{C}+\mathrm{CH} 3$ & 101.70 & 101.20 & 103.55 & 92.36 & 95.34 \\
\hline $\mathrm{Cl} 3 \mathrm{CCH} 3->\mathrm{Cl} 3 \mathrm{C}+\mathrm{CH} 3$ & 86.00 & 84.54 & 86.89 & 73.72 & 76.32 \\
\hline $\mathrm{CH} 2=\mathrm{CHC} 2 \mathrm{H} 5-->\mathrm{CH} 2=\mathrm{CH}+\mathrm{C} 2 \mathrm{H} 5$ & 100.50 & 98.84 & 100.64 & 89.01 & 91.57 \\
\hline $\mathrm{CH} 2=\mathrm{CHC} 2 \mathrm{H} 5 \rightarrow \mathrm{CH} 2=\mathrm{CHCH} 2+\mathrm{CH} 3$ & 75.90 & 73.70 & 76.33 & 64.52 & 66.60 \\
\hline $\mathrm{CH} 2=\mathrm{CHCH}(\mathrm{CH} 3) 2->\mathrm{CH} 2=\mathrm{CH}+\mathrm{CH}(\mathrm{CH} 3) 2$ & 100.00 & 97.02 & 98.74 & 84.41 & 87.59 \\
\hline $\mathrm{CH} 2=\mathrm{CHC} 3 \mathrm{H} 7-->\mathrm{CH} 2=\mathrm{CH}+\mathrm{n}-\mathrm{C} 3 \mathrm{H} 7$ & 100.50 & 99.22 & 100.97 & 89.13 & 91.75 \\
\hline $\mathrm{CH} 2=\mathrm{CHC} 3 \mathrm{H} 7-\rightarrow \mathrm{CH} 2=\mathrm{CHCH} 2+\mathrm{C} 2 \mathrm{H} 5$ & 74.80 & 72.46 & 75.01 & 60.88 & 63.52 \\
\hline $\mathrm{HCCC} 2 \mathrm{H} 5->\mathrm{CCH}+\mathrm{C} 2 \mathrm{H} 5$ & 124.50 & 125.56 & 126.75 & 118.61 & 120.81 \\
\hline $\mathrm{C} 6 \mathrm{H} 5 \mathrm{CHCH} 2->\mathrm{C} 6 \mathrm{H} 5+\mathrm{CHCH} 2$ & 117.70 & 115.32 & 116.68 & 104.88 & 107.97 \\
\hline $\mathrm{C} 6 \mathrm{H} 5 \mathrm{CN}->\mathrm{C} 6 \mathrm{H} 5+\mathrm{CN}$ & 133.80 & 132.75 & 134.40 & 127.13 & 132.85 \\
\hline $\mathrm{C} 6 \mathrm{H} 5 \mathrm{CHO}-->\mathrm{C} 6 \mathrm{H} 5+\mathrm{CHO}$ & 100.00 & 97.79 & 99.75 & 89.68 & 92.98 \\
\hline $\mathrm{C} 6 \mathrm{H} 5 \mathrm{COCH} 3 \rightarrow->\mathrm{C} 6 \mathrm{H} 5+\mathrm{COCH} 3$ & 99.50 & 96.08 & 98.14 & 84.80 & 88.79 \\
\hline $\mathrm{CH} 3 \mathrm{COCOCH} 3->2 \mathrm{CH} 3 \mathrm{CO}$ & 73.30 & 70.30 & 73.42 & 60.45 & 64.55 \\
\hline
\end{tabular}




\begin{tabular}{|c|c|c|c|c|c|}
\hline $\mathrm{C} 3 \mathrm{H} 7 \mathrm{COCH} 3-->n-\mathrm{C} 3 \mathrm{H} 7+\mathrm{COCH} 3$ & 83.30 & 81.15 & 83.54 & 70.78 & 74.28 \\
\hline $\mathrm{C} 6 \mathrm{H} 5 \mathrm{CH}(\mathrm{CH} 3) 2->\mathrm{C} 6 \mathrm{H} 5+\mathrm{CH}(\mathrm{CH} 3) 2$ & 101.74 & 98.94 & 100.55 & 84.52 & 88.12 \\
\hline $\mathrm{C} 6 \mathrm{H} 5 \mathrm{C} 6 \mathrm{H} 5$--> $2 \mathrm{C} 6 \mathrm{H} 5$ & 119.00 & 117.37 & 118.68 & 104.08 & 107.92 \\
\hline$n-\mathrm{C} 3 \mathrm{H} 7 \mathrm{NH} 2-->\mathrm{n}-\mathrm{C} 3 \mathrm{H} 7+\mathrm{NH} 2$ & 86.30 & 84.01 & 86.05 & 76.44 & 77.88 \\
\hline $\mathrm{i}-\mathrm{C} 3 \mathrm{H} 7 \mathrm{NH} 2-->\mathrm{i}-\mathrm{C} 3 \mathrm{H} 7+\mathrm{NH} 2$ & 86.60 & 83.89 & 85.88 & 74.51 & 76.27 \\
\hline $\mathrm{C} 2 \mathrm{H} 5 \mathrm{NO} 2$--> $\mathrm{C} 2 \mathrm{H} 5+\mathrm{NO} 2$ & 61.01 & 57.86 & 62.09 & 49.95 & 53.70 \\
\hline$n-\mathrm{C} 3 \mathrm{H} 7 \mathrm{NO} 2$--> n-C3H7 + NO2 & 61.61 & 58.00 & 62.20 & 49.74 & 53.58 \\
\hline i-C3H7NO2 -->i-C3H7 + NO2 & 62.91 & 58.36 & 62.44 & 47.93 & 52.19 \\
\hline $\mathrm{C} 6 \mathrm{H} 5 \mathrm{NO} 2$--> $66 \mathrm{H} 5+\mathrm{NO} 2$ & 72.91 & 70.23 & 74.17 & 62.08 & 66.30 \\
\hline $\mathrm{n}-\mathrm{C} 3 \mathrm{H} 7 \mathrm{SH}-->\mathrm{n}-\mathrm{C} 3 \mathrm{H} 7+\mathrm{SH}$ & 74.28 & 73.37 & 74.80 & 65.95 & 66.94 \\
\hline $\mathrm{i}-\mathrm{C} 3 \mathrm{H} 7 \mathrm{SH}-->\mathrm{i}-\mathrm{C} 3 \mathrm{H} 7+\mathrm{SH}$ & 73.88 & 71.95 & 73.35 & 62.48 & 63.84 \\
\hline $\mathrm{CH} 3 \mathrm{COSH}-->\mathrm{CH} 3 \mathrm{CO}+\mathrm{SH}$ & 73.58 & 72.72 & 74.05 & 67.62 & 68.19 \\
\hline $\mathrm{CH} 3 \mathrm{SC}_{2} \mathrm{H} 5-->\mathrm{CH} 3 \mathrm{~S}+\mathrm{C} 2 \mathrm{H} 5$ & 72.90 & 70.73 & 72.19 & 61.64 & 63.14 \\
\hline
\end{tabular}

\begin{tabular}{|c|c|c|c|c|c|c|}
\hline Methods & PBE & PBEO & M06-L & M06 & SCAN & SCANO \\
\hline $\mathrm{H}-\mathrm{OH}-->\mathrm{OH}+\mathrm{H}$ & 117.43 & 114.62 & 112.90 & 117.29 & 113.67 & 111.83 \\
\hline $\mathrm{H}-\mathrm{OCl}-->\mathrm{OCl}+\mathrm{H}$ & 89.06 & 90.53 & 88.71 & 94.06 & 89.60 & 89.98 \\
\hline $\mathrm{CH} 3 \mathrm{O}-\mathrm{H}-->\mathrm{CH} 3 \mathrm{O}+\mathrm{H}$ & 98.88 & 98.70 & 96.66 & 101.72 & 97.31 & 96.90 \\
\hline $\mathrm{C} 2 \mathrm{H} 5 \mathrm{O}-\mathrm{H}-->\mathrm{C} 2 \mathrm{H} 5 \mathrm{O}+\mathrm{H}$ & 98.17 & 98.43 & 96.60 & 101.74 & 97.17 & 96.94 \\
\hline $\mathrm{H}-\mathrm{SH}-->\mathrm{HS}+\mathrm{H}$ & 89.68 & 88.78 & 90.49 & 90.49 & 89.11 & 88.21 \\
\hline $\mathrm{CH} 3 \mathrm{~S}-\mathrm{H}-->\mathrm{CH} 3 \mathrm{~S}+\mathrm{H}$ & 83.68 & 83.41 & 84.85 & 85.38 & 83.77 & 83.19 \\
\hline $\mathrm{H}-\mathrm{NH} 2-->\mathrm{NH} 2+\mathrm{H}$ & 105.22 & 103.92 & 102.32 & 105.68 & 102.10 & 101.55 \\
\hline $\mathrm{H}-\mathrm{NH}-->\mathrm{NH}+\mathrm{H}$ & 94.16 & 91.99 & 92.08 & 92.78 & 93.61 & 91.66 \\
\hline $\mathrm{H}-\mathrm{PH} 2-->\mathrm{PH} 2+\mathrm{H}$ & 79.63 & 79.29 & 82.28 & 82.66 & 79.96 & 79.35 \\
\hline $\mathrm{CH} 3-\mathrm{H}-->\mathrm{CH} 3+\mathrm{H}$ & 102.43 & 101.71 & 103.70 & 104.49 & 99.50 & 99.40 \\
\hline $\mathrm{CH} 2-\mathrm{H}->\mathrm{CH} 2(3 \mathrm{~B} 1)+\mathrm{H}$ & 109.06 & 108.33 & 108.26 & 108.81 & 109.21 & 108.47 \\
\hline $\mathrm{CH} 2(3 \mathrm{~B} 1)-->\mathrm{CH}+\mathrm{H}$ & 104.65 & 105.54 & 104.55 & 102.73 & 109.65 & 109.77 \\
\hline $\mathrm{C} 2 \mathrm{H} 5-\mathrm{H}-->\mathrm{C} 2 \mathrm{H} 5+\mathrm{H}$ & 97.11 & 97.04 & 98.15 & 99.48 & 94.95 & 95.13 \\
\hline$(\mathrm{CH} 3) 2 \mathrm{CH}-\mathrm{H}-->(\mathrm{CH} 3) 2 \mathrm{CH}+\mathrm{H}$ & 92.92 & 93.34 & 93.73 & 95.60 & 91.57 & 91.91 \\
\hline$(\mathrm{CH} 3) 3 \mathrm{C}-\mathrm{H}-->(\mathrm{CH} 3) 3 \mathrm{C}+\mathrm{H}$ & 89.89 & 90.69 & 90.45 & 93.04 & 89.35 & 89.78 \\
\hline $\mathrm{CH} 2=\mathrm{CH}-\mathrm{H}-->\mathrm{CH} 2 \mathrm{CH}+\mathrm{H}$ & 106.20 & 106.63 & 106.13 & 108.02 & 104.55 & 105.02 \\
\hline $\mathrm{HCC}-\mathrm{H}-->\mathrm{CCH}+\mathrm{H}$ & 131.23 & 131.10 & 127.37 & 130.75 & 128.93 & 128.85 \\
\hline $\mathrm{C} 6 \mathrm{H} 5-\mathrm{H}->\mathrm{C} 6 \mathrm{H} 5+\mathrm{H}$ & 107.95 & 108.85 & 107.39 & 109.89 & 106.64 & 107.13 \\
\hline $\mathrm{CH} 3 \mathrm{OH}-->\mathrm{CH} 2 \mathrm{OH}+\mathrm{H}$ & 91.29 & 91.91 & 94.13 & 95.39 & 90.81 & 91.07 \\
\hline $\mathrm{H}-\mathrm{CN}-->\mathrm{CN}+\mathrm{H}$ & 123.22 & 126.82 & 122.13 & 126.49 & 124.11 & 126.56 \\
\hline $\mathrm{H}-\mathrm{CF} 3-->\mathrm{CF} 3+\mathrm{H}$ & 100.91 & 101.99 & 101.83 & 105.11 & 101.14 & 101.38 \\
\hline $\mathrm{H} 2 \mathrm{CO}-->\mathrm{HCO}+\mathrm{H}$ & 84.26 & 85.31 & 86.23 & 88.56 & 84.52 & 85.16 \\
\hline $\mathrm{H}-\mathrm{CO}-->\mathrm{CO}+\mathrm{H}$ & 22.61 & 21.42 & 19.23 & 17.53 & 24.01 & 23.19 \\
\hline $\mathrm{CH} 3 \mathrm{CHO}-->\mathrm{CH} 3 \mathrm{CO}+\mathrm{H}$ & 84.96 & 86.10 & 86.86 & 89.21 & 85.53 & 86.07 \\
\hline $\mathrm{CH} 3-\mathrm{O}-->\mathrm{CH} 3+\mathrm{O}$ & 100.30 & 92.13 & 92.05 & 92.36 & 91.94 & 85.88 \\
\hline $\mathrm{C} 2 \mathrm{H} 5-\mathrm{O}-->\mathrm{C} 2 \mathrm{H} 5+\mathrm{O}$ & 100.93 & 92.87 & 91.74 & 92.60 & 93.38 & 87.20 \\
\hline $\mathrm{CH} 3-\mathrm{S}->\mathrm{CH} 3+\mathrm{S}$ & 76.31 & 73.07 & 72.78 & 72.36 & 70.48 & 68.27 \\
\hline CF3-F --> CF3 $+F$ & 129.62 & 125.80 & 125.64 & 131.12 & 124.60 & 120.66 \\
\hline $\mathrm{CH} 2=\mathrm{CH}-\mathrm{F}-->\mathrm{CH} 2 \mathrm{CH}+\mathrm{F}$ & 127.99 & 121.63 & 120.14 & 123.70 & 120.03 & 114.74 \\
\hline
\end{tabular}




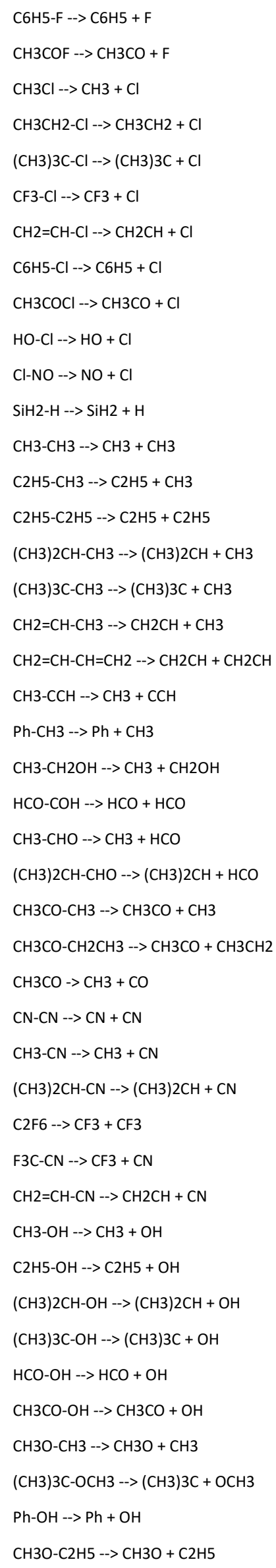

\begin{tabular}{|c|c|c|c|c|c|}
\hline 29.97 & 124.12 & 122.31 & 126.37 & 122.79 & 117.41 \\
\hline 25.02 & 119.61 & 120.03 & 123.73 & 119.77 & 114.75 \\
\hline 5.31 & 82.29 & 84.53 & 83.66 & 78.79 & 76.6 \\
\hline 4.25 & 81.68 & 83.20 & 83.01 & 79.11 & 76.91 \\
\hline 80.65 & 78.84 & 80.33 & 81.90 & 78.56 & 76.11 \\
\hline 36.13 & 83.85 & 85.79 & 86.48 & 83.10 & 80.26 \\
\hline 5.80 & 93.08 & 93.80 & 92.99 & 90.44 & 88.13 \\
\hline 96.76 & 94.51 & 94.70 & 94.60 & 92.23 & 89.77 \\
\hline 6.57 & 83.37 & 87.29 & 86.36 & 83.82 & 80.46 \\
\hline 62.17 & 53.21 & 53.81 & 54.13 & 52.25 & 45.63 \\
\hline 51.08 & 38.17 & 48.27 & 41.94 & 44.26 & 32.91 \\
\hline 86.52 & 87.12 & 91.56 & 93.28 & 87.85 & 87.97 \\
\hline 89.82 & 88.20 & 89.27 & 89.66 & 81.11 & 80.88 \\
\hline 6.42 & 85.43 & 85.55 & 86.84 & 79.00 & 78.90 \\
\hline 82.98 & 82.62 & 81.86 & 84.04 & 76.84 & 76.89 \\
\hline 83.27 & 82.78 & 82.30 & 84.62 & 77.19 & 77.14 \\
\hline 79.99 & 79.99 & 79.49 & 82.98 & 75.44 & 75.36 \\
\hline 99.46 & 98.77 & 97.63 & 98.86 & 92.25 & 92.33 \\
\hline 12.92 & 112.67 & 110.02 & 111.29 & 107.40 & 107.26 \\
\hline 28.50 & 127.26 & 123.44 & 125.54 & 120.07 & 119.7 \\
\hline 100.05 & 99.83 & 97.86 & 99.98 & 93.49 & 93.51 \\
\hline 83.91 & 83.54 & 84.88 & 85.83 & 78.27 & 78.17 \\
\hline 68.46 & 69.59 & 68.99 & 71.29 & 66.68 & 67.04 \\
\hline 83.88 & 83.69 & 83.55 & 85.12 & 78.77 & 78.89 \\
\hline 76.28 & 77.20 & 75.39 & 78.67 & 73.65 & 73.96 \\
\hline 81.70 & 81.96 & 81.96 & 83.95 & 77.42 & 77.61 \\
\hline 78.33 & 79.28 & 78.88 & 81.53 & 75.57 & 75.87 \\
\hline 21.52 & 19.00 & 15.92 & 13.43 & 17.25 & 16.02 \\
\hline 140.98 & 145.03 & 137.66 & 141.04 & 138.60 & 140.97 \\
\hline 23.56 & 126.19 & 121.52 & 124.48 & 118.56 & 120.87 \\
\hline 91 & 119.69 & 113.11 & 117.45 & 113.37 & 115.88 \\
\hline 3.66 & 90.41 & 91.98 & 96.04 & 89.31 & 88.60 \\
\hline 10.05 & 112.99 & 108.88 & 113.25 & 108.41 & 109.54 \\
\hline 31.55 & 134.42 & 128.38 & 131.29 & 127.99 & 129.95 \\
\hline 93.78 & 89.50 & 88.99 & 92.28 & 85.25 & 82.38 \\
\hline 93.69 & 89.97 & 88.63 & 92.54 & 86.56 & 83.73 \\
\hline 3.10 & 89.78 & 87.82 & 92.51 & 87.33 & 84.45 \\
\hline 1.04 & 88.96 & 87.00 & 92.56 & 87.57 & 84.64 \\
\hline 112.03 & 108.83 & 107.69 & 111.55 & 107.42 & 104.64 \\
\hline 110.16 & 107.74 & 106.98 & 111.11 & 106.58 & 104.07 \\
\hline .13 & 78.37 & 77.36 & 81.88 & 74.20 & 72.62 \\
\hline 74.30 & 74.00 & 73.09 & 79.43 & 72.90 & 71.26 \\
\hline 2.69 & 109.23 & 106.68 & 110.70 & 106.57 & 103.35 \\
\hline 9.95 & 78.77 & 00 & 82.12 & .42 & 7 \\
\hline
\end{tabular}




\begin{tabular}{|c|c|c|c|c|c|c|}
\hline $\mathrm{CH} 3-\mathrm{OC} 2 \mathrm{H} 5$-> CH3 + OC2H5 & 79.32 & 78.02 & 77.31 & 81.88 & 73.98 & 72.59 \\
\hline $\mathrm{C} 2 \mathrm{H} 5-\mathrm{OC} 2 \mathrm{H} 5-\rightarrow \mathrm{C} 2 \mathrm{H} 5+\mathrm{OC} 2 \mathrm{H} 5$ & 79.04 & 78.34 & 76.91 & 82.07 & 75.11 & 73.79 \\
\hline $\mathrm{CH} 3 \mathrm{CO}-\mathrm{OCH} 3-\rightarrow \mathrm{CH} 3 \mathrm{CO}+\mathrm{OCH}$ & 94.67 & 94.86 & 94.26 & 99.05 & 94.03 & 2.75 \\
\hline $\mathrm{HCO}-\mathrm{OCH} 3$--> HCO + OCH3 & 97.10 & 96.47 & 95.48 & 99.95 & 95.40 & 3.8 \\
\hline $\mathrm{CH} 3-\mathrm{NH} 2-\rightarrow \mathrm{CH} 3+\mathrm{NH} 2$ & 84.94 & 82.54 & 80.79 & 83.78 & 76.32 & 75.35 \\
\hline $\mathrm{C} 2 \mathrm{H} 5-\mathrm{NH} 2-\rightarrow \mathrm{C} 2 \mathrm{H} 5+\mathrm{NH} 2$ & 83.64 & 81.71 & 78.81 & 82.64 & 76.28 & 75.3 \\
\hline $\mathrm{Ph}-\mathrm{NH} 2$--> Ph + NH2 & 103.92 & 102.40 & 98.27 & 102.24 & 97.83 & 96.5 \\
\hline (CH3)3C-NH2 --> (CH3)3C $+\mathrm{NH}_{2}$ & 79.67 & 78.76 & 75.49 & 81.25 & 75.39 & 74.42 \\
\hline $\mathrm{CH} 3 \mathrm{CO}-\mathrm{NH} 2->\mathrm{CH} 3 \mathrm{CO}+\mathrm{NH} 2$ & 98.22 & 97.62 & 94.98 & 99.09 & 94.76 & 93.9 \\
\hline $\mathrm{CH} 3-\mathrm{NO} 2-\rightarrow \mathrm{CH} 3+\mathrm{NO} 2$ & 57.11 & 58.01 & 53.94 & 58.60 & 53.54 & 54.28 \\
\hline $\mathrm{CH} 3-\mathrm{SH}-->\mathrm{CH} 3+\mathrm{SH}$ & 75.50 & 73.22 & 74.22 & 73.93 & 68.98 & 67.6 \\
\hline $\mathrm{C} 2 \mathrm{H} 5-\mathrm{SH}-->\mathrm{C} 2 \mathrm{H} 5+\mathrm{SH}$ & 72.49 & 70.75 & 70.76 & 71.37 & 67.26 & 65.93 \\
\hline$(\mathrm{CH} 3) 3 \mathrm{C}-\mathrm{SH}-->(\mathrm{CH} 3) 3 \mathrm{C}+\mathrm{SH}$ & 67.23 & 66.20 & 65.77 & 68.63 & 64.78 & 63.2 \\
\hline CH3S-CH3 - -> CH3S + CH3 & 72.28 & 70.55 & 71.29 & 71.64 & 66.68 & 65.5 \\
\hline $\mathrm{HO}-\mathrm{OH}-->\mathrm{OH}+\mathrm{OH}$ & 57.73 & 47.08 & 46.06 & 47.57 & 45.52 & 37.64 \\
\hline $\mathrm{H} 2 \mathrm{~N}-\mathrm{NH} 2-\rightarrow \mathrm{NH} 2+\mathrm{NH} 2$ & 68.27 & 64.53 & 59.95 & 64.89 & 58.26 & 56.4 \\
\hline $\mathrm{CH} 3 \mathrm{O}-\mathrm{NO} \rightarrow-\mathrm{CH} 3 \mathrm{O}+\mathrm{NO}$ & 48.47 & 39.79 & 42.73 & 42.57 & 41.67 & 34.0 \\
\hline $\mathrm{CH} 3-\mathrm{SiH} 3-\rightarrow \mathrm{CH} 3+\mathrm{SiH} 3$ & 85.07 & 84.82 & 87.83 & 88.26 & 81.30 & 81.09 \\
\hline SiH3-SiH3 $->\mathrm{SiH} 3+\mathrm{SiH} 3$ & 72.43 & 72.91 & 76.66 & 76.63 & 71.92 & 71.5 \\
\hline $\mathrm{HCCl} 3 \rightarrow \mathrm{CCl} 3+\mathrm{H}$ & 87.18 & 88.24 & 87.77 & 91.27 & 87.22 & 87.6 \\
\hline HONO --> H + NO2 & 70.40 & 72.41 & 69.15 & 75.59 & 72.37 & 73.78 \\
\hline HONO2 --> H + NO3 & 88.42 & 98.42 & 91.35 & 102.37 & 92.93 & 101 \\
\hline$n-C 3 H 7 F-\rightarrow n-C 3 H 7+F$ & 115.28 & 109.10 & 108.56 & 112.26 & 107.19 & 102 \\
\hline $\mathrm{ClCN}-\rightarrow \mathrm{Cl}+\mathrm{CN}$ & 106.73 & 106.33 & 105.00 & 105.44 & 102.07 & 101 \\
\hline $\mathrm{CCl} 4->\mathrm{CCl} 3+\mathrm{Cl}$ & 65.94 & 62.90 & 63.69 & 66.25 & 61.80 & 58.7 \\
\hline $\mathrm{i}-\mathrm{C} 3 \mathrm{H} 7 \mathrm{Cl}-->\mathrm{i}-\mathrm{C} 3 \mathrm{H} 7+\mathrm{Cl}$ & 82.71 & 80.53 & 81.78 & 82.38 & 79.03 & 76.7 \\
\hline $\mathrm{C} 2 \mathrm{H} 5 \mathrm{OOOC} 2 \mathrm{H} 5$--> $2 \mathrm{C} 2 \mathrm{H} 5 \mathrm{O}$ & 33.65 & 28.23 & 27.27 & 30.01 & 28.02 & 22.3 \\
\hline $\mathrm{NO} 3$--> NO2 + $\mathrm{O}$ & 71.15 & 52.32 & 55.61 & 50.47 & 59.18 & 42.8 \\
\hline HONO --> HO + NO & 60.93 & 49.90 & 52.44 & 52.12 & 51.46 & 42.6 \\
\hline HONO2 --> HO + NO2 & 54.17 & 49.41 & 47.23 & 51.04 & 48.12 & 44 \\
\hline $\mathrm{C} 2 \mathrm{H} 5 \mathrm{ONO}-->\mathrm{C} 2 \mathrm{H} 5 \mathrm{O}+\mathrm{NO}$ & 46.53 & 38.55 & 41.16 & 41.59 & 40.29 & 32.9 \\
\hline $\mathrm{C} 2 \mathrm{H} 5 \mathrm{NO} 3$--> $22 \mathrm{H} 5 \mathrm{O}+\mathrm{NO} 2$ & 40.26 & 38.20 & 36.79 & 40.74 & 37.73 & 34.88 \\
\hline HSSH --> 2 HS & 67.06 & 63.41 & 65.91 & 65.27 & 62.19 & 59.2 \\
\hline $\mathrm{CH} 3 \mathrm{SSCH}_{3}-->2 \mathrm{CH} 3 \mathrm{~S}$ & 63.40 & 60.44 & 62.64 & 62.91 & 60.30 & 57. \\
\hline $\mathrm{n}-\mathrm{C} 3 \mathrm{H} 7 \mathrm{NO3}-->\mathrm{n}-\mathrm{C} 3 \mathrm{H} 7+\mathrm{NO} 3$ & 69.90 & 78.73 & 73.13 & 83.09 & 71.97 & 79.2 \\
\hline $\mathrm{i}-\mathrm{C} 3 \mathrm{H} 7 \mathrm{NO} 3$--> i-C3H7 + NO3 & 68.64 & 77.82 & 71.95 & 82.41 & 72.03 & 79.2 \\
\hline $\mathrm{C} 2 \mathrm{H} 5 \mathrm{NO} 33->\mathrm{C} 2 \mathrm{H} 5+\mathrm{NO} 3$ & 70.04 & 78.76 & 72.93 & 82.88 & 71.93 & 79.2 \\
\hline $\mathrm{n}-\mathrm{C} 5 \mathrm{H} 12-->\mathrm{n}-\mathrm{C} 3 \mathrm{H} 7+\mathrm{C} 2 \mathrm{H} 5$ & 83.04 & 82.73 & 82.25 & 84.46 & 77.10 & 77.8 \\
\hline n-C6H14 --> 2 n-C3H7 & 83.18 & 82.93 & 82.63 & 84.87 & 77.37 & 77.4 \\
\hline $\mathrm{C} 2 \mathrm{H} 5 \mathrm{CH}(\mathrm{CH} 3) 2->\mathrm{C} 2 \mathrm{H} 5+\mathrm{CH}(\mathrm{CH} 3) 2$ & 78.93 & 79.06 & 78.23 & 81.38 & 74.22 & 74.2 \\
\hline $\mathrm{C} 2 \mathrm{H} 5 \mathrm{CH}(\mathrm{CH} 3) 2-\mathrm{CH} 3+\mathrm{CH} 2 \mathrm{CH}(\mathrm{CH} 3) 2$ & 86.09 & 85.16 & 86.14 & 87.48 & 78.99 & 78.8 \\
\hline$(\mathrm{CH} 3)_{2} \mathrm{CHC}(\mathrm{CH} 3)_{3}-->(\mathrm{CH} 3) 2 \mathrm{CH}+\mathrm{C}(\mathrm{CH} 3)_{3}$ & 68.70 & 69.81 & 70.39 & 75.18 & 67.28 & 67.3 \\
\hline$(\mathrm{CH} 3)_{2} \mathrm{CH}(\mathrm{CH} 2) 2 \mathrm{OH}->(\mathrm{CH} 3) 2 \mathrm{CHCH} 2+\mathrm{CH} 2 \mathrm{OH}$ & 79.78 & 80.12 & 81.82 & 83.60 & 75.90 & 75.9 \\
\hline $3 \mathrm{CCH} 3->\mathrm{F} 3 \mathrm{C}+\mathrm{CH} 3$ & 98.36 & 98.69 & 98.63 & 101.33 & 94.52 & \\
\hline
\end{tabular}




\begin{tabular}{|c|c|c|c|c|c|c|}
\hline $\mathrm{Cl} 3 \mathrm{CCH} 3-->\mathrm{Cl} 3 \mathrm{C}+\mathrm{CH} 3$ & 80.27 & 80.36 & 80.02 & 83.97 & 76.52 & 76.15 \\
\hline $\mathrm{CH} 2=\mathrm{CHC} 2 \mathrm{H} 5->\mathrm{CH} 2=\mathrm{CH}+\mathrm{C} 2 \mathrm{H} 5$ & 95.60 & 95.52 & 93.33 & 95.43 & 89.61 & 89.83 \\
\hline $\mathrm{CH} 2=\mathrm{CHC} 2 \mathrm{H} 5->\mathrm{CH} 2=\mathrm{CHCH} 2+\mathrm{CH} 3$ & 71.56 & 70.92 & 70.66 & 72.20 & 62.58 & 62.69 \\
\hline $\mathrm{CH} 2=\mathrm{CHCH}(\mathrm{CH} 3) 2->\mathrm{CH} 2=\mathrm{CH}+\mathrm{CH}(\mathrm{CH} 3) 2$ & 91.57 & 92.06 & 89.87 & 92.75 & 87.15 & 87.43 \\
\hline $\mathrm{CH} 2=\mathrm{CHC} 3 \mathrm{H} 7-->\mathrm{CH} 2=\mathrm{CH}+n-\mathrm{C} 3 \mathrm{H} 7$ & 95.82 & 95.79 & 93.81 & 95.94 & 89.99 & 90.22 \\
\hline $\mathrm{CH} 2=\mathrm{CHC} 3 \mathrm{H} 7->\mathrm{CH} 2=\mathrm{CHCH} 2+\mathrm{C} 2 \mathrm{H} 5$ & 68.14 & 68.13 & 67.02 & 69.43 & 60.47 & 60.71 \\
\hline $\mathrm{HCCC} 2 \mathrm{H} 5-\rightarrow \mathrm{CCH}+\mathrm{C} 2 \mathrm{H} 5$ & 124.47 & 123.83 & 118.89 & 121.72 & 117.23 & 117.07 \\
\hline $\mathrm{C} 6 \mathrm{H} 5 \mathrm{CHCH} 2-\rightarrow \mathrm{C} 6 \mathrm{H} 5+\mathrm{CHCH} 2$ & 111.42 & 111.79 & 108.88 & 110.91 & 106.63 & 106.56 \\
\hline $\mathrm{C} 6 \mathrm{H} 5 \mathrm{CN} \rightarrow-\mathrm{C} 6 \mathrm{H} 5+\mathrm{CN}$ & 132.93 & 136.36 & 129.49 & 133.13 & 130.08 & 132.01 \\
\hline $\mathrm{C} 6 \mathrm{H} 5 \mathrm{CHO}-->\mathrm{C} 6 \mathrm{H} 5+\mathrm{CHO}$ & 96.20 & 97.02 & 95.05 & 97.50 & 93.67 & 93.53 \\
\hline $\mathrm{C} 6 \mathrm{H} 5 \mathrm{COCH} 3-\rightarrow \mathrm{C} 6 \mathrm{H} 5+\mathrm{COCH} 3$ & 92.06 & 93.52 & 92.14 & 94.83 & 90.56 & 90.61 \\
\hline $\mathrm{CH} 3 \mathrm{COCOCH} 3$--> $2 \mathrm{CH} 3 \mathrm{CO}$ & 67.94 & 69.78 & 70.22 & 72.92 & 68.22 & 68.45 \\
\hline $\mathrm{C} 3 \mathrm{H} 7 \mathrm{COCH} 3-->\mathrm{n}-\mathrm{C} 3 \mathrm{H} 7+\mathrm{COCH} 3$ & 78.29 & 79.29 & 79.10 & 81.75 & 75.67 & 75.99 \\
\hline $\mathrm{C} 6 \mathrm{H} 5 \mathrm{CH}(\mathrm{CH} 3) 2->\mathrm{C} 6 \mathrm{H} 5+\mathrm{CH}(\mathrm{CH} 3) 2$ & 91.75 & 92.69 & 89.87 & 93.79 & 88.09 & 88.25 \\
\hline $\mathrm{C} 6 \mathrm{H} 5 \mathrm{C} 6 \mathrm{H} 5$--> $2 \mathrm{C} 6 \mathrm{H} 5$ & 111.02 & 112.16 & 108.26 & 111.56 & 107.22 & 107.27 \\
\hline $\mathrm{n}-\mathrm{C} 3 \mathrm{H} 7 \mathrm{NH} 2-->\mathrm{n}-\mathrm{C} 3 \mathrm{H} 7+\mathrm{NH} 2$ & 83.47 & 81.73 & 79.27 & 83.10 & 76.36 & 75.50 \\
\hline $\mathrm{i}-\mathrm{C} 3 \mathrm{H} 7 \mathrm{NH2}-->\mathrm{i}-\mathrm{C} 3 \mathrm{H} 7+\mathrm{NH} 2$ & 81.68 & 80.29 & 76.94 & 81.67 & 75.79 & 74.87 \\
\hline $\mathrm{C} 2 \mathrm{H} 5 \mathrm{NO} 2->\mathrm{C} 2 \mathrm{H} 5+\mathrm{NO} 2$ & 56.09 & 57.54 & 53.43 & 58.45 & 54.01 & 54.76 \\
\hline $\mathrm{n}-\mathrm{C} 3 \mathrm{H} 7 \mathrm{NO2} 2-\rightarrow \mathrm{n}-\mathrm{C} 3 \mathrm{H} 7+\mathrm{NO2}$ & 55.74 & 57.33 & 52.93 & 58.11 & 53.78 & 54.61 \\
\hline i-C3H7NO2 -->i-C3H7 + NO2 & 54.10 & 56.13 & 51.52 & 57.31 & 53.53 & 54.35 \\
\hline $\mathrm{C} 6 \mathrm{H} 5 \mathrm{NO2} 2->\mathrm{C} 6 \mathrm{H} 5+\mathrm{NO} 2$ & 68.26 & 70.07 & 65.23 & 70.07 & 67.32 & 67.67 \\
\hline$n-C 3 H 7 S H-->n-C 3 H 7+S H$ & 73.39 & 71.61 & 71.87 & 72.48 & 68.24 & 66.87 \\
\hline $\mathrm{i}-\mathrm{C} 3 \mathrm{H} 7 \mathrm{SH}-\rightarrow \mathrm{i}-\mathrm{C} 3 \mathrm{H} 7+\mathrm{SH}$ & 70.12 & 68.73 & 68.23 & 69.89 & 66.21 & 64.80 \\
\hline $\mathrm{CH} 3 \mathrm{COSH}-->\mathrm{CH} 3 \mathrm{CO}+\mathrm{SH}$ & 75.75 & 73.40 & 75.31 & 75.04 & 73.14 & 70.61 \\
\hline $\mathrm{CH} 3 \mathrm{SC}_{2} \mathrm{H} 5 \mathrm{-}-\mathrm{CH} 3 \mathrm{~S}+\mathrm{C} 2 \mathrm{H} 5$ & 69.09 & 67.90 & 67.71 & 68.98 & 64.78 & 63.67 \\
\hline
\end{tabular}


Table S7. Optimized heights of Carbon Atom to $\mathrm{NaCl}(100)$ Surface (dZ, in $\AA$, in C-down configuration), calculated adsorption energies $\left(E_{a}\right.$ in $\mathrm{kJ} / \mathrm{mol}$ ), and the frequencies (in $\left.\mathrm{cm}^{-1}\right)$.

\begin{tabular}{lccccc}
\hline Methods & $\mathrm{dZ}$ & $E_{a}$ & $F_{c o}{ }^{\mathrm{a}}$ & $F_{C-\text { down }^{\mathrm{a}}}$ & $F_{O-\text { down }^{\mathrm{a}}}$ \\
\hline BLYP & 2.723 & 4.96 & 2015 & 2017 & 2016 \\
PBE & 2.659 & 11.63 & 2026 & 2030 & 2024 \\
M06-L & 2.529 & 27.60 & 2099 & 2101 & 2093 \\
SCAN & 2.515 & 19.12 & 2103 & 2102 & 2092 \\
& & & & & \\
B3LYP & 2.701 & 7.20 & 2108 & 2114 & 2110 \\
PBE0 & 2.626 & 11.35 & 2132 & 2139 & 2131 \\
M06 & 2.595 & 24.31 & 2132 & 2138 & 2116 \\
& & & & & \\
XYG3 & 2.591 & $21.28^{\mathrm{c}}$ & 2016 & 2021 & 2008 \\
XYGJ-OS & 2.592 & 18.94 & 2032 & 2040 & 2026 \\
& & & & & \\
Expt. $^{\mathrm{b}}$ & 2.590 & 18.00 & 2041 & 2051 & 2034 \\
\hline
\end{tabular}

a): $F_{c o}$ is the calculated $\mathrm{CO}$ vibrational frequency on a free $\mathrm{CO}$ monolayer on the lattice of 5.64 $\AA$ and vacuum of $30 \AA$ along the $\mathrm{Z}$-direction. $F_{C \text {-down }}$ is the calculated $\mathrm{CO}$ vibrational frequency on the C-down adsorption configuration. $F_{O-\text { down }}$ is the calculated $\mathrm{CO}$ vibrational frequency on the O-down adsorption configuration.

b): The experimental dZ, adsorption energy, and frequence are taken from References ${ }^{22},{ }^{23}$, and ${ }^{28}$,respectively.

c) XYG3 adsorption energy was obtained with NAO-VCC-3Z (N3Z) and corrected by the XYGJ-OS basis set error at N3Z, i.e. $E_{a}$ [CBS, XYGOS]- $E_{a}[\mathrm{~N} 3 Z, \mathrm{XYGOS}$ ]. (refer to Table S10 for this correction). 
Table S8. Optimized heights of Carbon Atom to $\mathrm{NaCl}(100)$ Surface (dZ, in $\AA$, in C-down configuration) by using numerical force (dZ@NF) with displacement of 0.001 and by using analytic force (dZ@AF).

\begin{tabular}{lcc}
\hline Methods & dZ@NF & dZ@AF \\
\hline PBE & 2.659 & 2.658 \\
PBE0 & 2.626 & 2.628 \\
M06 & 2.610 & 2.607 \\
& & \\
Expt. $^{a}$ & 2.590 & \\
\hline
\end{tabular}

a): The experimental $\mathrm{dZ}$ was taken from References ${ }^{22}$. 
Table S9. CO vibrational frequency on the C-down adsorption configuration $\left(F_{C-\text { down }}\right.$ in $\mathrm{cm}^{-1}$ )_obtained by using numerical force with displacement of $0.001 \AA$ (NF01), $0.005 \AA$ (NF02), $0.010 \AA$ (NF03), $0.013 \AA$ (NF04), and $0.015 \AA$ (NF05), respectively.

\begin{tabular}{lccccc}
\hline Methods & NF01 & NF02 & NF03 & NF04 & NF05 \\
\hline PBE & 2031 & 2029 & 2030 & 2030 & 2030 \\
Literature $^{\mathrm{a}}$ & 2032 & & & & \\
Expt. $^{\mathrm{b}}$ & 2051 & & & & \\
\hline
\end{tabular}

a): Planewave PBE result by the VASP code ${ }^{21}$, which employed the same two-layer rigid $\mathrm{NaCl}(100)$ model of the experimental lattice and a vacuum of $15 \AA$. Projector-augmented wave potentials were employed to treat the core electrons. The numerical hessian was obtained with analytic forces.

b): The experimental frequency was taken from References ${ }^{28}$. 
Table S10. Adsorption energies of XYGJ-OS ( $E_{a}$ in $\left.\mathrm{kJ} / \mathrm{mol}\right)$ by using different basis set and the extrapolated values in the complete-basis-set (CBS) limit

\begin{tabular}{lc}
\hline Basis Set & $E_{a}$ \\
\hline NAO-VCC-3Z & -21.7 \\
NAO-VCC-4Z & -20.1 \\
CBS[34] & -18.9 \\
Expt. $^{a}$ & -18.0 \\
\hline
\end{tabular}

a): The experimental adsorption energy was taken from References ${ }^{22}$. 
Geom1: Optimized coordinates for main-group cubic crystals (Unit in $\AA$; Format in FHI-aims)

\section{1) C and Si with diamond structure}

lattice_vector $0.000 \quad<a 2>\quad<a 2>$
lattice_vector $<a 2>0.000 \quad<a 2>$
lattice_vector $<a 2>\quad<a 2>0.000$
atom_frac $0.0000 .0000 .000<$ atom01>
atom_frac $0.5000 .5000 .500<$ atom01>
where:
$<a 2>=$ half of the lattice constant
$<$ atom01> = the species name, i.e. "C" or "Si"

2) LiH, LiF, LiCl,NaF, NaCl, MgO, and MgS with rocksalt structure

\begin{tabular}{|c|c|c|c|}
\hline lattice_vector & 0.000 & $<\mathrm{a} 2>$ & $<a 2>$ \\
\hline lattice_vector & $<a 2>$ & 0.000 & $<a 2>$ \\
\hline lattice_vector & $<a 2>$ & $<a 2>$ & 0.000 \\
\hline
\end{tabular}

where:

$<$ a2 $>=$ half of the lattice constant

$<$ atom01> = the species name of the metal element, i.e. " $\mathrm{Li}$ ", "Na", or " $\mathrm{Mg}$ "

$<$ atom02> = the species name of the non-metal element, i.e. " $\mathrm{H}$ ", " $\mathrm{F}$ ", " $\mathrm{Cl}$ ", "O", or " $\mathrm{S}$ "

\section{3) BeS, BN, BP, SiC, and AIP with zincblende structure}

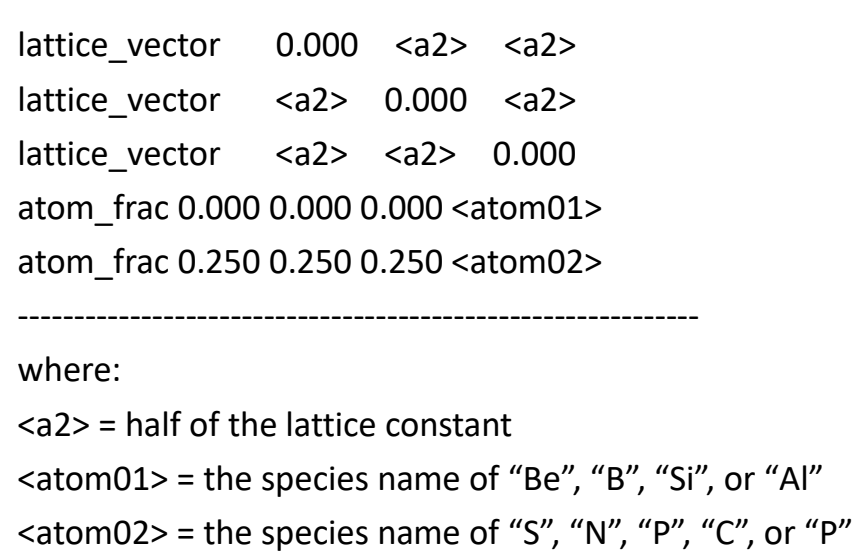




\section{4) Ne and Ar with fcc structure}

lattice_vector $0.000<\mathrm{a} 2\rangle \quad\langle\mathrm{a} 2\rangle$

lattice_vector <a2> $0.000<\mathrm{a} 2>$

lattice_vector $<\mathrm{a} 2>\quad<\mathrm{a} 2>0.000$

atom_frac 0.0000 .0000 .000 <atom01>

where:

$<a 2>=$ half of the lattice constant

$<$ atom01> = the species name, i.e. "Ne" or "Ar" 
Geom-2: Optimized coordinates for Rutile TiO2 by PBE (Unit in Å; Format in FHI-aims)

1) Volume $=57.60 \AA^{3}$

\begin{tabular}{|c|c|c|c|}
\hline lattice_vector & 4.45470000 & 0.00000000 & 0.00000000 \\
\hline lattice_vector & 0.00000000 & 4.45470000 & 0.00000000 \\
\hline lattice_vector & 0.00000000 & 0.00000000 & 2.90250000 \\
\hline atom_frac & 0.00000000 & 0.00000000 & $0.00000000 \mathrm{Ti}$ \\
\hline \multicolumn{4}{|c|}{ constrain_relaxation .true. } \\
\hline atom_frac & 0.50000000 & 0.50000000 & $0.50000000 \mathrm{Ti}$ \\
\hline \multicolumn{4}{|c|}{ constrain_relaxation .true. } \\
\hline atom_frac & 0.30227649 & 0.30227629 & 0.000000000 \\
\hline \multicolumn{4}{|c|}{ constrain_relaxation z } \\
\hline atom_frac & 0.69772351 & 0.69772371 & 0.000000000 \\
\hline \multicolumn{4}{|c|}{ constrain_relaxation z } \\
\hline atom_frac & 0.19772352 & 0.80227627 & 0.500000000 \\
\hline \multicolumn{4}{|c|}{ constrain_relaxation z } \\
\hline atom_frac & 0.80227648 & 0.19772373 & 0.500000000 \\
\hline - & on z & & \\
\hline
\end{tabular}

2) Volume $=57.733 \AA^{3}$

\begin{tabular}{|c|c|c|c|}
\hline lattice_vector & 4.51830000 & 0.00000000 & 0.00000000 \\
\hline lattice_vector & 0.00000000 & 4.51830000 & 0.00000000 \\
\hline lattice_vector & 0.00000000 & 0.00000000 & 2.92590000 \\
\hline atom_frac & 0.00000000 & 0.00000000 & $0.00000000 \mathrm{Ti}$ \\
\hline \multicolumn{4}{|c|}{ constrain_relaxation .true. } \\
\hline atom_frac & 0.50000000 & 0.50000000 & $0.50000000 \mathrm{Ti}$ \\
\hline \multicolumn{4}{|c|}{ constrain_relaxation .true. } \\
\hline atom_frac & 0.30298017 & 0.30297993 & 0.000000000 \\
\hline \multicolumn{4}{|c|}{ constrain_relaxation z } \\
\hline atom_frac & 0.69701983 & 0.69702007 & 0.000000000 \\
\hline \multicolumn{4}{|c|}{ constrain_relaxation z } \\
\hline atom_frac & 0.19701982 & 0.80297993 & 0.500000000 \\
\hline \multicolumn{4}{|c|}{ constrain_relaxation z } \\
\hline $\begin{array}{l}\text { atom_frac } \\
\text { constrain_rela }\end{array}$ & $\begin{array}{l}0.80298018 \\
\text { kation z }\end{array}$ & 0.19702007 & 0.500000000 \\
\hline
\end{tabular}

\section{3) Volume $=61.867 \AA^{3}$}

$\begin{array}{llll}\text { lattice_vector } & 4.58180000 & 0.00000000 & 0.00000000 \\ \text { lattice_vector } & 0.00000000 & 4.58180000 & 0.00000000\end{array}$




\begin{tabular}{|c|c|c|c|}
\hline lattice_vector & 0.00000000 & 0.00000000 & 2.94700000 \\
\hline atom_frac & 0.00000000 & 0.00000000 & $0.00000000 \mathrm{Ti}$ \\
\hline \multicolumn{4}{|c|}{ constrain_relaxation .true. } \\
\hline atom_frac & 0.50000000 & 0.50000000 & $0.50000000 \mathrm{Ti}$ \\
\hline \multicolumn{4}{|c|}{ constrain_relaxation .true. } \\
\hline atom_frac & 0.30395766 & 0.30395821 & 0.000000000 \\
\hline \multicolumn{4}{|c|}{ constrain_relaxation z } \\
\hline atom_frac & 0.69604234 & 0.69604179 & 0.000000000 \\
\hline \multicolumn{4}{|c|}{ constrain_relaxation z } \\
\hline atom_frac & 0.19604233 & 0.80395822 & 0.500000000 \\
\hline \multicolumn{4}{|c|}{ constrain_relaxation z } \\
\hline atom_frac & 0.80395767 & 0.19604177 & 0.500000000 \\
\hline \multicolumn{4}{|c|}{ constrain_relaxation z } \\
\hline
\end{tabular}

\section{4) Volume $=63.841 \AA^{3}$}

\begin{tabular}{|c|c|c|c|c|}
\hline lattice_vector & 4.64020000 & 0.00000000 & \multicolumn{2}{|l|}{0.00000000} \\
\hline lattice_vector & 0.00000000 & 4.64020000 & \multicolumn{2}{|l|}{0.00000000} \\
\hline lattice_vector & 0.00000000 & 0.00000000 & \multicolumn{2}{|l|}{2.96500000} \\
\hline atom_frac & 0.00000000 & 0.00000000 & 0.00000000 & $\mathrm{Ti}$ \\
\hline \multicolumn{5}{|c|}{ constrain_relaxation .true. } \\
\hline atom_frac & 0.50000000 & 0.50000000 & 0.50000000 & $\mathrm{Ti}$ \\
\hline \multicolumn{5}{|c|}{ constrain_relaxation .true. } \\
\hline atom_frac & 0.30510000 & 0.30510000 & 0.00000000 & $\mathrm{O}$ \\
\hline \multicolumn{5}{|c|}{ constrain_relaxation z } \\
\hline atom_frac & 0.69490000 & 0.69490000 & 0.00000000 & $\mathrm{O}$ \\
\hline \multicolumn{5}{|c|}{ constrain_relaxation z } \\
\hline atom_frac & 0.19490000 & 0.80510000 & 0.50000000 & $\mathrm{O}$ \\
\hline \multicolumn{5}{|c|}{ constrain_relaxation z } \\
\hline atom_frac & 0.80510000 & 0.19490000 & 0.5000000 & $\mathrm{O}$ \\
\hline constrain_re & on z & & & \\
\hline
\end{tabular}

\section{5) Volume $=64.000 \AA^{3}$}

\begin{tabular}{|c|c|c|c|}
\hline lattice_vector & 4.64580000 & 0.00000000 & 0.00000000 \\
\hline lattice_vector & 0.00000000 & 4.64580000 & 0.00000000 \\
\hline lattice_vector & 0.00000000 & 0.00000000 & 2.96520000 \\
\hline atom_frac & 0.00000000 & 0.00000000 & $0.00000000 \mathrm{Ti}$ \\
\hline \multicolumn{4}{|c|}{ constrain_relaxation .true. } \\
\hline atom_frac & 0.50000000 & 0.50000000 & $0.50000000 \mathrm{Ti}$ \\
\hline \multicolumn{4}{|c|}{ constrain_relaxation .true. } \\
\hline atom_frac & 0.30518660 & 0.30518662 & 0.000000000 \\
\hline
\end{tabular}


constrain_relaxation z

$\begin{array}{ccc}\begin{array}{l}\text { atom_frac } \quad 0.69481341 \\ \text { constrain_relaxation z }\end{array} & 0.69481337 & 0.000000000 \\ \begin{array}{l}\text { atom_frac } \quad 0.19481340 \\ \text { constrain_relaxation z }\end{array} & 0.80518662 & 0.500000000 \\ \begin{array}{l}\text { atom_frac } 0.80518659 \\ \text { constrain_relaxation z }\end{array} & 0.19481337 & 0.500000000\end{array}$

6) Volume $=66.133 \AA^{3}$

\begin{tabular}{|c|c|c|c|}
\hline lattice_vector & 4.71080000 & 0.00000000 & 0.00000000 \\
\hline lattice_vector & 0.00000000 & 4.71080000 & 0.00000000 \\
\hline lattice_vector & 0.00000000 & 0.00000000 & 2.98000000 \\
\hline atom_frac & 0.00000000 & 0.00000000 & $0.00000000 \mathrm{Ti}$ \\
\hline \multicolumn{4}{|c|}{ constrain_relaxation .true. } \\
\hline atom_frac & 0.50000000 & 0.50000000 & $0.50000000 \mathrm{Ti}$ \\
\hline \multicolumn{4}{|c|}{ constrain_relaxation .true. } \\
\hline atom_frac & 0.30686017 & 0.30686033 & 0.000000000 \\
\hline \multicolumn{4}{|c|}{ constrain_relaxation z } \\
\hline atom_frac & 0.69313984 & 0.69313965 & 0.000000000 \\
\hline \multicolumn{4}{|c|}{ constrain_relaxation z } \\
\hline atom_frac & 0.19313984 & 0.80686035 & 0.500000000 \\
\hline \multicolumn{4}{|c|}{ constrain_relaxation z } \\
\hline atom_frac & 0.80686017 & 0.19313967 & 0.500000000 \\
\hline
\end{tabular}

\section{7) Volume $=70.400 \AA^{3}$}

\begin{tabular}{|c|c|c|c|}
\hline lattice_vector & 4.84650000 & 0.00000000 & 0.00000000 \\
\hline lattice_vector & 0.00000000 & 4.84650000 & 0.00000000 \\
\hline lattice_vector & 0.00000000 & 0.00000000 & 2.99720000 \\
\hline atom_frac & 0.00000000 & 0.00000000 & $0.00000000 \mathrm{Ti}$ \\
\hline \multicolumn{4}{|c|}{ constrain_relaxation .true. } \\
\hline atom_frac & 0.50000000 & 0.50000000 & $0.50000000 \mathrm{Ti}$ \\
\hline \multicolumn{4}{|c|}{ constrain_relaxation .true. } \\
\hline atom_frac & 0.31102096 & 0.31102078 & 0.00000000 \\
\hline \multicolumn{4}{|c|}{ constrain_relaxation z } \\
\hline atom_frac & 0.68897907 & 0.68897919 & 0.00000000 \\
\hline \multicolumn{4}{|c|}{ constrain_relaxation z } \\
\hline atom_frac & 0.18897906 & 0.81102078 & 0.50000000 \\
\hline \multicolumn{4}{|c|}{ constrain_relaxation z } \\
\hline atom_frac & 0.81102093 & 0.18897920 & 0.50000000 \\
\hline
\end{tabular}


constrain_relaxation z 


\section{Geom3: Optimized coordinates for Rutile TiO2 by SCAN (Unit in Å; Format in FHI-aims)}

1) Volume $=57.60 \AA^{3}$

\begin{tabular}{|c|c|c|c|}
\hline \\
\hline lattice_vector & 4.45300000 & 0.00000000 & 0.00000000 \\
\hline lattice_vector & 0.00000000 & 4.45300000 & 0.00000000 \\
\hline lattice_vector & 0.00000000 & 0.00000000 & 2.90480000 \\
\hline atom_frac & 0.00000000 & 0.00000000 & $0.00000000 \mathrm{Ti}$ \\
\hline \multicolumn{4}{|c|}{ constrain_relaxation .true. } \\
\hline atom_frac & 0.50000000 & 0.50000000 & $0.50000000 \mathrm{Ti}$ \\
\hline \multicolumn{4}{|c|}{ constrain_relaxation .true. } \\
\hline atom_frac & 0.30295614 & 0.30295558 & 0.000000000 \\
\hline \multicolumn{4}{|c|}{ constrain_relaxation z } \\
\hline atom_frac & 0.69704386 & 0.69704442 & 0.000000000 \\
\hline \multicolumn{4}{|c|}{ constrain_relaxation z } \\
\hline atom_frac & 0.19704387 & 0.80295558 & 0.500000000 \\
\hline \multicolumn{4}{|c|}{ constrain_relaxation z } \\
\hline atom_frac & 0.80295614 & 0.19704442 & 0.500000000 \\
\hline
\end{tabular}

\section{2) Volume $=59.733 \AA^{3}$}

\begin{tabular}{|c|c|c|c|}
\hline lattice_vector & 4.51690000 & 0.00000000 & 0.00000000 \\
\hline lattice_vector & 0.00000000 & 4.51690000 & 0.00000000 \\
\hline lattice_vector & 0.00000000 & 0.00000000 & 2.92780000 \\
\hline $\begin{array}{l}\text { atom_frac } \\
\text { constrain_re }\end{array}$ & $\begin{array}{l}0.00000000 \\
\text { ation .true. }\end{array}$ & 0.00000000 & $0.00000000 \mathrm{Ti}$ \\
\hline \multicolumn{4}{|c|}{ constrain_relaxation .true. } \\
\hline $\begin{array}{l}\text { atom_frac } \\
\text { constrain_re }\end{array}$ & $\begin{array}{l}0.30370311 \\
\text { ation z }\end{array}$ & 0.30370310 & 0.000000000 \\
\hline $\begin{array}{l}\text { atom_frac } \\
\text { constrain_re }\end{array}$ & \multicolumn{2}{|c|}{ constrain_relaxation z } & 0.000000000 \\
\hline \multicolumn{4}{|c|}{ constrain_relaxation z } \\
\hline $\begin{array}{l}\text { atom_frac } \\
\text { constrain_re }\end{array}$ & $\begin{array}{l}0.80370311 \\
\text { ation z }\end{array}$ & 0.19629690 & 0.500000000 \\
\hline
\end{tabular}

\section{3) Volume $=61.897 \AA^{3}$}

$\begin{array}{llll}\text { lattice_vector } & 4.58060000 & 0.00000000 & 0.00000000 \\ \text { lattice_vector } & 0.00000000 & 4.58060000 & 0.00000000 \\ \text { lattice_vector } & 0.00000000 & 0.00000000 & 2.94860000\end{array}$




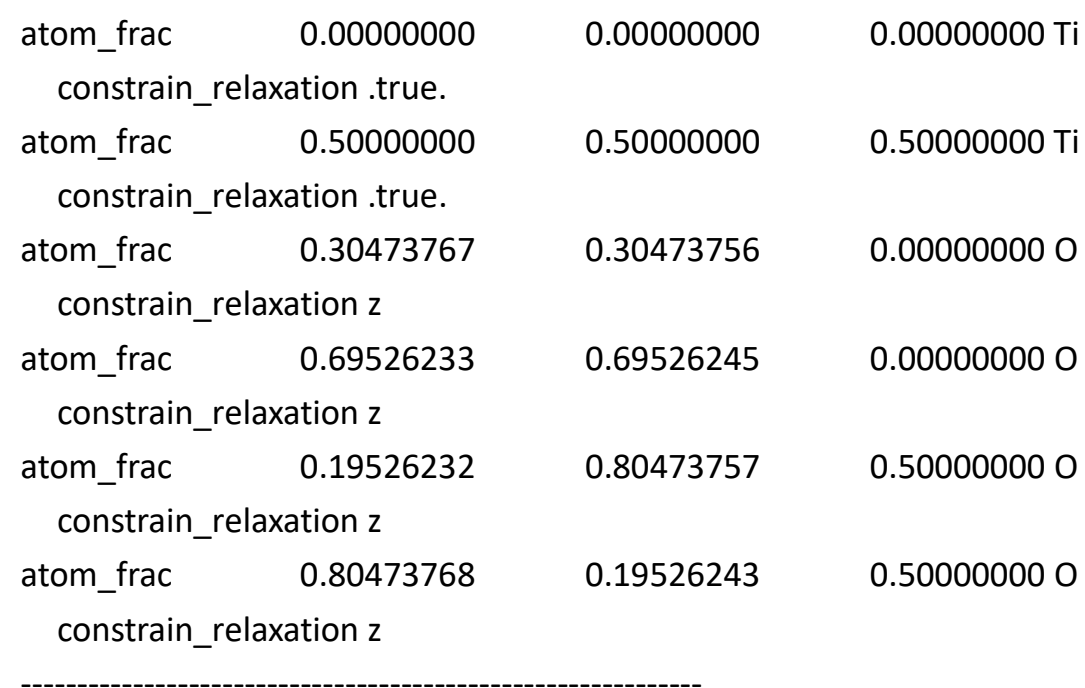

\section{4) Volume $=64.000 \AA^{3}$}

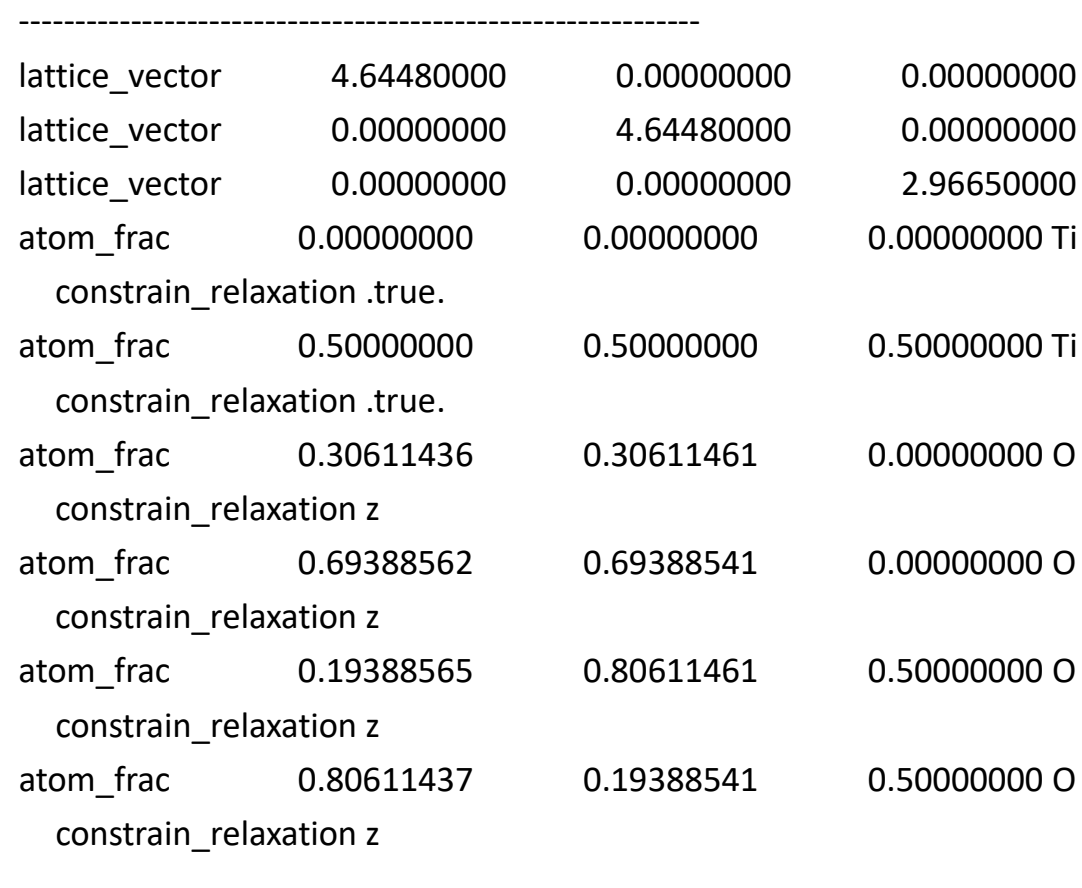

\section{5) Volume $=66.133 \AA^{3}$}

\begin{tabular}{|c|c|c|c|}
\hline lattice_vector & 4.71030000 & 0.00000000 & 0.00000000 \\
\hline lattice_vector & 0.00000000 & 4.71030000 & 0.00000000 \\
\hline lattice_vector & 0.00000000 & 0.00000000 & 2.98080000 \\
\hline \multicolumn{4}{|c|}{ constrain_relaxation .true. } \\
\hline $\begin{array}{l}\text { atom_frac } \\
\text { constrain_rel }\end{array}$ & $\begin{array}{l}0.50000000 \\
\text { ation .true. }\end{array}$ & 0.50000000 & $0.50000000 \mathrm{Ti}$ \\
\hline $\begin{array}{l}\text { atom_frac } \\
\text { constrain_rel }\end{array}$ & $\begin{array}{l}0.30778770 \\
\text { ation z }\end{array}$ & 0.30778786 & 0.000000000 \\
\hline
\end{tabular}




\begin{tabular}{cccc} 
atom_frac & 0.69221229 & 0.69221215 & 0.000000000 \\
constrain_relaxation z & & \\
atom_frac $\quad 0.19221230$ & 0.80778786 & 0.500000000 \\
\multicolumn{2}{c}{ constrain_relaxation z } & & \\
atom_frac 0.80778771 & 0.19221215 & 0.500000000 \\
constrain_relaxation z
\end{tabular}

6) Volume $=68.267 \AA^{3}$

\begin{tabular}{|c|c|c|c|}
\hline lattice_vector & 4.77800000 & 0.00000000 & 0.00000000 \\
\hline lattice_vector & 0.00000000 & 4.77800000 & 0.00000000 \\
\hline lattice_vector & 0.00000000 & 0.00000000 & 2.99030000 \\
\hline \multicolumn{4}{|c|}{ constrain_relaxation .true. } \\
\hline atom_frac & 0.50000000 & 0.50000000 & $0.50000000 \mathrm{Ti}$ \\
\hline \multicolumn{4}{|c|}{ constrain_relaxation .true. } \\
\hline \multicolumn{4}{|c|}{ constrain_relaxation z } \\
\hline \multicolumn{4}{|c|}{ constrain_relaxation z } \\
\hline $\begin{array}{l}\text { atom_frac } \\
\text { constrain_re }\end{array}$ & $\begin{array}{l}0.19019030 \\
\text { ation z }\end{array}$ & 0.80980971 & 0.500000000 \\
\hline $\begin{array}{l}\text { atom_frac } \\
\text { constrain }\end{array}$ & $\begin{array}{l}0.80980969 \\
\text { ation z }\end{array}$ & 0.19019028 & 0.500000000 \\
\hline
\end{tabular}

\section{7) Volume $=70.400 \AA^{3}$}

\begin{tabular}{|c|c|c|c|}
\hline lattice_vector & 4.84830000 & 0.00000000 & 0.00000000 \\
\hline lattice_vector & 0.00000000 & 4.84830000 & 0.00000000 \\
\hline lattice_vector & 0.00000000 & 0.00000000 & 2.99500000 \\
\hline atom_frac & 0.00000000 & 0.00000000 & $0.00000000 \mathrm{Ti}$ \\
\hline \multicolumn{4}{|c|}{ constrain_relaxation .true. } \\
\hline atom_frac & 0.50000000 & 0.50000000 & $0.50000000 \mathrm{Ti}$ \\
\hline \multicolumn{4}{|c|}{ constrain_relaxation .true. } \\
\hline atom_frac & 0.31233560 & 0.31233562 & 0.000000000 \\
\hline \multicolumn{4}{|c|}{ constrain_relaxation z } \\
\hline $\begin{array}{l}\text { atom_frac } \\
\text { constrain_re }\end{array}$ & $\begin{array}{l}0.68766444 \\
\text { ation z }\end{array}$ & 0.68766433 & 0.000000000 \\
\hline atom_frac & 0.18766439 & 0.81233564 & 0.500000000 \\
\hline \multicolumn{4}{|c|}{ constrain_relaxation z } \\
\hline atom_frac & 0.81233559 & 0.18766432 & 0.50000000 \\
\hline
\end{tabular}


constrain_relaxation z 
Geom-4: Optimized coordinates for Anatase TiO2 by PBE (Unit in Å; Format in FHI-aims)

1) Volume $=61.312 \AA^{3}$

lattice_vector $\quad-1.869750 \quad 1.869750 \quad 4.384450$

lattice_vector $\quad 1.869750-1.869750 \quad 4.384450$

lattice_vector $\quad 1.869750 \quad 1.869750-4.384450$

atom_frac $\quad 0.125000 \quad 0.875000 \quad 0.250000 \quad \mathrm{Ti}$

constrain_relaxation .true.

atom_frac $\quad 0.875000 \quad 0.125000 \quad 0.750000 \quad \mathrm{Ti}$

constrain_relaxation .true.

atom_frac 0.3454210 .0954210 .2500000

constrain_relaxation $z$

atom_frac $\quad 0.0954210 .3454210 .7500000$

constrain_relaxation z

atom_frac $\quad 0.6545790 .9045790 .7500000$

constrain_relaxation z

atom_frac $\quad 0.9045790 .6545790 .2500000$

constrain_relaxation $\mathrm{z}$

\section{2) Volume $=63.585 \AA^{3}$}

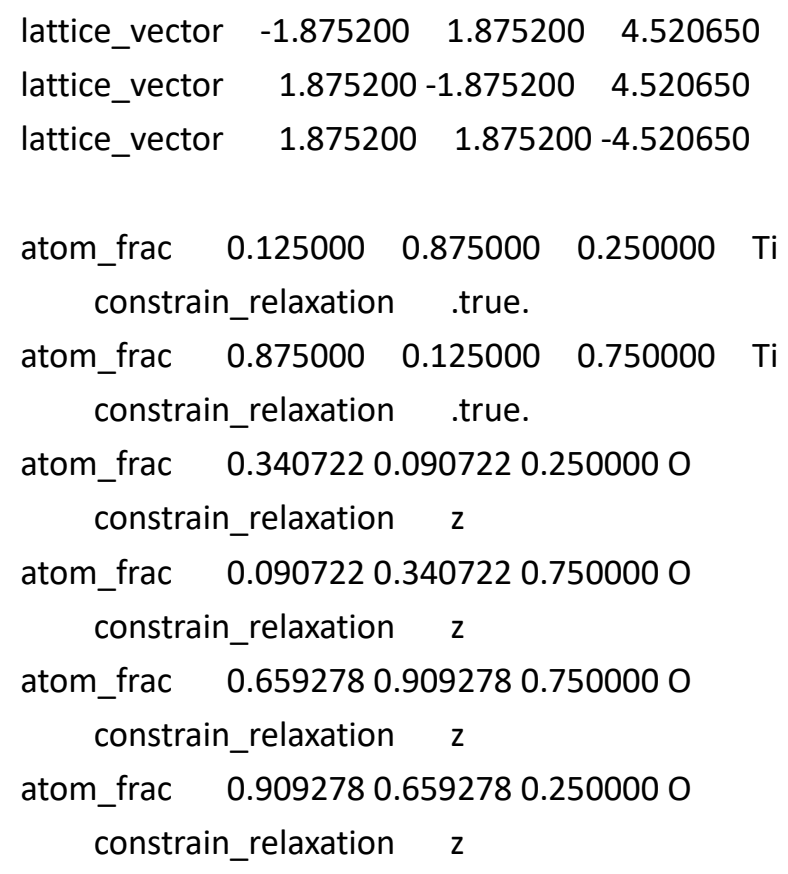

3) Volume $=65.856 \AA^{3}$ 


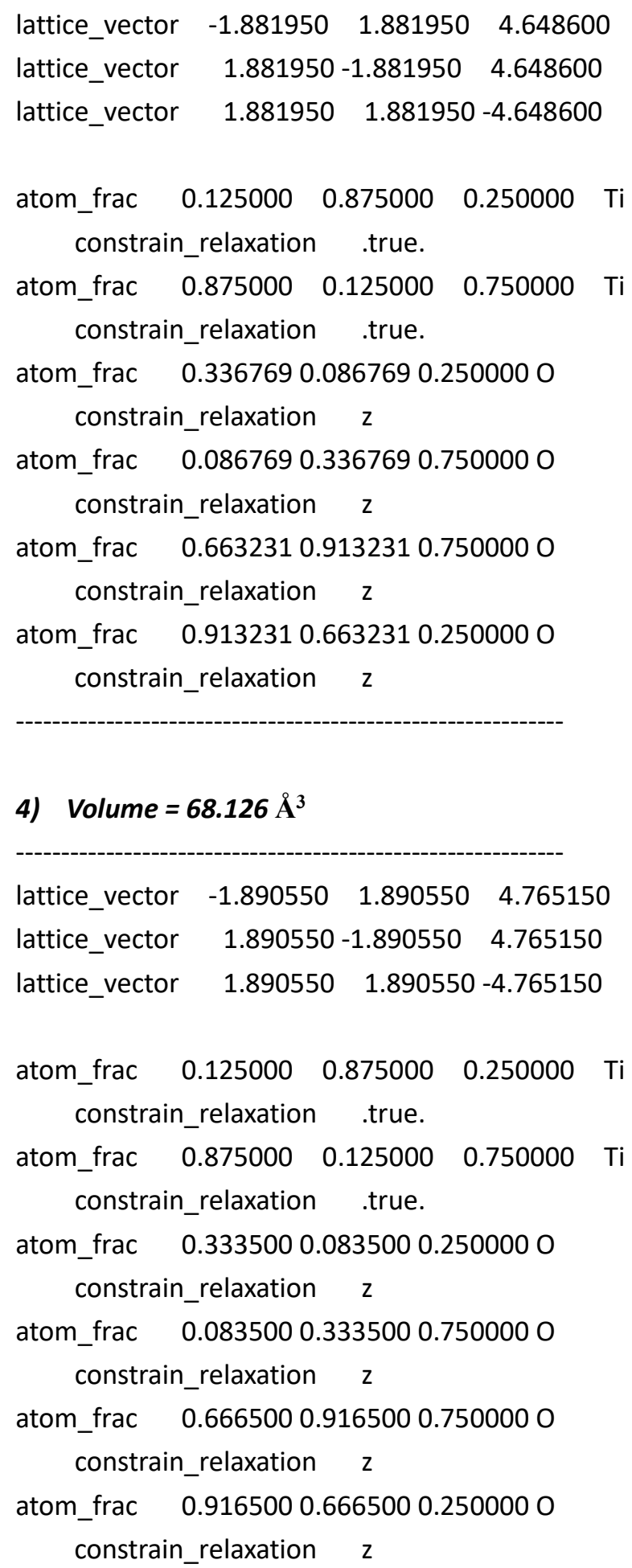

5) Volume $=70.398 \AA^{3}$

lattice_vector $\quad-1.901150 \quad 1.901150 \quad 4.869300$

lattice_vector $\quad 1.901150-1.901150 \quad 4.869300$

lattice_vector $\quad 1.901150 \quad 1.901150-4.869300$ 
atom_frac $\quad 0.3272130 .0772130 .2500000$ constrain_relaxation z

atom_frac $\quad 0.0772130 .3272130 .7500000$ constrain_relaxation z

atom_frac $\quad 0.6727870 .9227870 .7500000$ constrain_relaxation z

atom_frac $\quad 0.9227870 .6727870 .2500000$ constrain_relaxation $\mathrm{z}$ 
Geom5: Optimized coordinates for Anatase TiO2 by SCAN (Unit in $\AA$; Format in FHI-aims)

1) Volume $=61.311 \AA^{3}$

lattice_vector $\quad-1.860500 \quad 1.860500 \quad 4.428150$

lattice_vector $\quad 1.860500-1.860500 \quad 4.428150$

lattice_vector $\quad \begin{array}{lll}1.860500 & 1.860500-4.428150\end{array}$

atom_frac $\quad 0.125000 \quad 0.875000 \quad 0.250000 \quad \mathrm{Ti}$

constrain_relaxation .true.

atom_frac $\quad 0.875000 \quad 0.125000 \quad 0.750000 \quad \mathrm{Ti}$

constrain_relaxation true.

atom_frac $\quad 0.3420470 .0920470 .2500000$

constrain_relaxation z

atom_frac $\quad 0.0920470 .3420470 .7500000$

constrain_relaxation $z$

atom_frac $\quad 0.6579530 .9079530 .7500000$

constrain_relaxation z

atom_frac $\quad 0.9079530 .6579530 .2500000$

constrain_relaxation $\mathrm{z}$

2) Volume $=63.584 \AA^{3}$

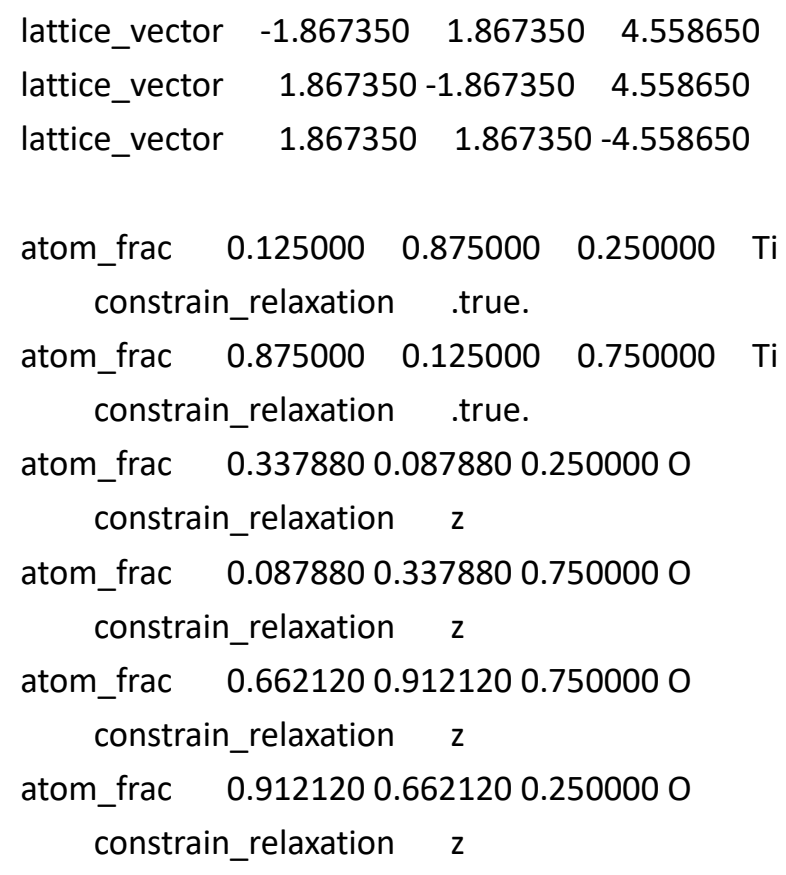

3) Volume $=65.854 \AA^{3}$ 


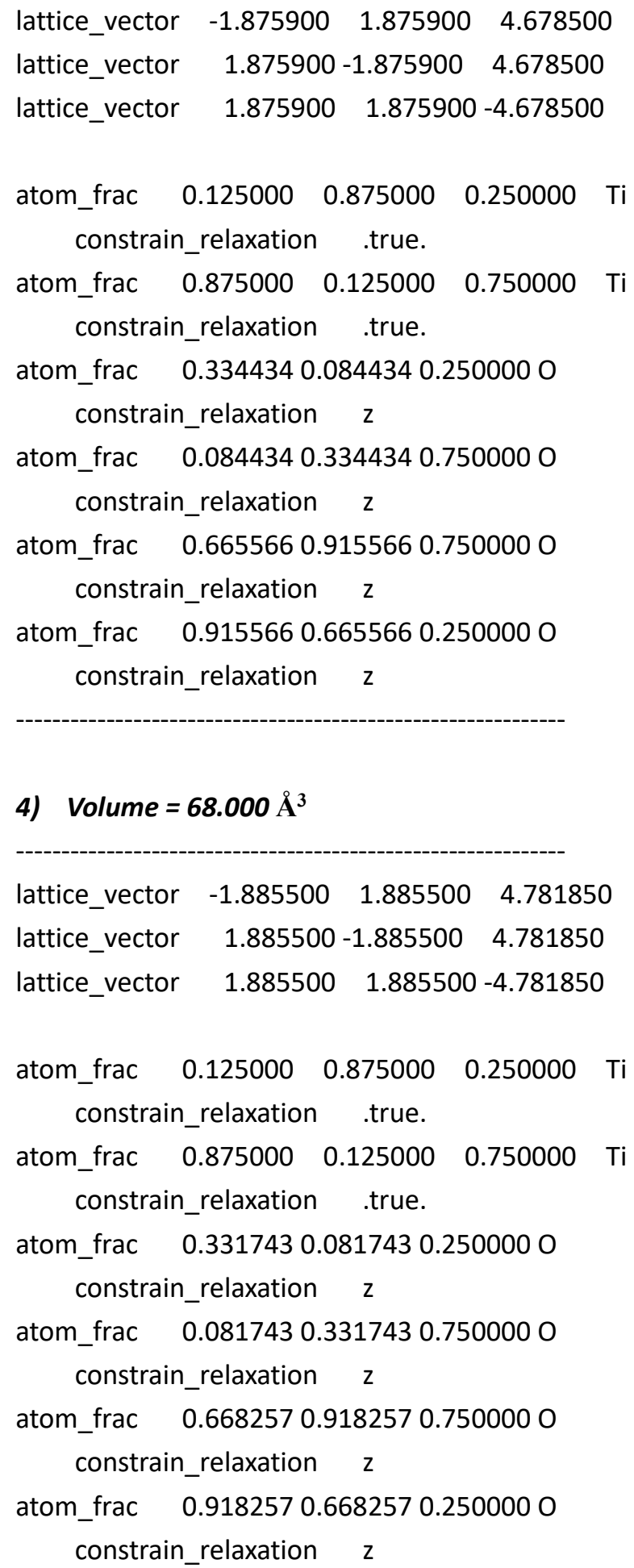

5) Volume $=70.397 \AA^{3}$

lattice_vector $\quad-1.898650 \quad 1.898650 \quad 4.882050$

lattice_vector $\quad 1.898650-1.898650 \quad 4.882050$

lattice_vector $\quad 1.898650 \quad 1.898650-4.882050$ 
atom_frac $\quad 0.3260120 .0760120 .2500000$ constrain_relaxation z

atom_frac $\quad 0.0760120 .3260120 .7500000$ constrain_relaxation z

atom_frac $\quad 0.6739880 .9239880 .7500000$ constrain_relaxation z

atom_frac $\quad 0.9239880 .6739880 .2500000$ constrain_relaxation

Z 
Geom6: Optimized coordinates for $\mathrm{CO}$ adsorption on a rigid $\mathrm{NaCl}(100)$ surface with C-down configuration by XYGJ-OS (Unit in Å; Format in FHI-aims)

\begin{tabular}{|c|c|c|c|c|}
\hline lattice_vector & \multicolumn{2}{|c|}{5.64000000} & 0.00000000 & 0.00000000 \\
\hline lattice_vector & \multicolumn{2}{|c|}{2.82000000} & 2.82000000 & 0.00000000 \\
\hline lattice_vector & \multicolumn{2}{|c|}{0.00000000} & & 30.00000000 \\
\hline atom & 0.00000000 & 0.00000000 & \multicolumn{2}{|c|}{$15.00000000 \mathrm{Na}$} \\
\hline \multicolumn{5}{|c|}{ constrain_relaxation .true. } \\
\hline atom & 0.00000000 & 0.00000000 & \multicolumn{2}{|c|}{$17.82000000 \mathrm{Cl}$} \\
\hline \multicolumn{5}{|c|}{ constrain_relaxation .true. } \\
\hline atom & 2.82000000 & 0.00000000 & \multicolumn{2}{|c|}{$17.82000000 \mathrm{Na}$} \\
\hline \multicolumn{5}{|c|}{ constrain_relaxation .true. } \\
\hline atom & 2.82000000 & 0.00000000 & \multicolumn{2}{|c|}{$15.00000000 \mathrm{Cl}$} \\
\hline \multicolumn{5}{|c|}{ constrain_relaxation .true. } \\
\hline atom & 3.82889009 & -1.01160031 & & $73 \mathrm{O}$ \\
\hline atom & 3.34678358 & -0.52455358 & & $58 \mathrm{C}$ \\
\hline
\end{tabular}

Geom7: Optimized coordinates for $\mathrm{CO}$ adsorption on a rigid $\mathrm{NaCl}(100)$ surface with O-down configuration by XYGJ-OS (Unit in Å; Format in FHI-aims)

\begin{tabular}{|c|c|c|c|}
\hline lattice_vector & \multirow{2}{*}{$\begin{array}{l}5.64000000 \\
2.82000000\end{array}$} & \multicolumn{2}{|c|}{0.00000000} \\
\hline lattice_vector & & \multicolumn{2}{|c|}{2.82000000} \\
\hline lattice_vector & 0.00000000 & 0.00000000 & \\
\hline atom & 0.00000000 & 0.00000000 & $15.00000000 \mathrm{Na}$ \\
\hline \multicolumn{4}{|c|}{ constrain_relaxation .true. } \\
\hline atom & 0.00000000 & 0.00000000 & $17.82000000 \mathrm{Cl}$ \\
\hline \multicolumn{4}{|c|}{ constrain_relaxation .true. } \\
\hline atom & 2.82000000 & \multirow[t]{2}{*}{0.00000000} & \multirow[t]{2}{*}{$17.82000000 \mathrm{Na}$} \\
\hline \multicolumn{2}{|c|}{ constrain_relaxation .true. } & & \\
\hline atom & 2.82000000 & 0.00000000 & $15.00000000 \mathrm{Cl}$ \\
\hline \multicolumn{4}{|c|}{ constrain_relaxation .true. } \\
\hline atom & 3.82889009 & -1.01160031 & 21.310206730 \\
\hline atom & 3.34678358 & -0.52455358 & $20.41206858 \mathrm{C}$ \\
\hline
\end{tabular}

Geom8: Optimized coordinates for free $\mathrm{CO}$ monolayer with the experimental lattice of $\mathbf{5 . 6 4}$ $\AA ̊$ and a vacuum of $30.0 \AA ̊$ by XYGJ-OS (Unit in $\AA$; Format in FHI-aims)

\begin{tabular}{llll}
\hline lattice_vector & 5.64000000 & 0.00000000 & 0.000000000 \\
lattice_vector & 2.82000000 & 2.82000000 & 0.000000000 \\
lattice_vector & 0.00000000 & 0.00000000 & 30.000000000 \\
atom & 3.89093513 & -1.06509534 & 21.303174030 \\
atom & 3.30341779 & -0.49508464 & $20.52314330 \mathrm{C}$
\end{tabular}


Geom9: Two-layer rigid $\mathrm{NaCl}(100)$ slide (Unit in Å; Format in FHI-aims)

\begin{tabular}{lrcr}
\hline lattice_vector & 5.64000000 & 0.00000000 & 0.00000000 \\
lattice_vector & 2.82000000 & 2.82000000 & 0.00000000 \\
lattice_vector & 0.00000000 & 0.00000000 & 30.00000000 \\
atom & 0.00000000 & 0.00000000 & $15.000000000 \mathrm{Na}$ \\
constrain_relaxation .true. & & \\
atom & 0.00000000 & 0.00000000 & $17.82000000 \mathrm{Cl}$ \\
constrain_relaxation .true. & & \\
atom & 2.82000000 & 0.00000000 & $17.82000000 \mathrm{Na}$ \\
constrain_relaxation .true. & & \\
atom 2.82000000 & 0.00000000 & $15.00000000 \mathrm{Cl}$ \\
constrain_relaxation .true. & &
\end{tabular}

